\title{
Cultivation-independent and cultivation- dependent metagenomes reveal genetic and enzymatic potential of microbial community involved in the degradation of a complex microbial polymer
}

\author{
Ohana Y. A. Costa ${ }^{1,2}$, Mattias de Hollander ${ }^{1}$, Agata Pijl', Binbin Liu ${ }^{3^{*}}$ and Eiko E. Kuramae ${ }^{1^{*}}$
}

\begin{abstract}
Background: Cultivation-independent methods, including metagenomics, are tools for the exploration and discovery of biotechnological compounds produced by microbes in natural environments. Glycoside hydrolases (GHs) enzymes are extremely desired and important in the industry of production for goods and biofuel and removal of problematic biofilms and exopolysaccharide (EPS). Biofilms and EPS are complex, requiring a wide range of enzymes for a complete degradation. The aim of this study was to identify potential GH microbial producers and GH genes with biotechnological potential, using EPS-complex structure (WH15EPS) of Acidobacteria Granulicella sp. strain WH15 as an enrichment factor, in cultivation-independent and cultivation-dependent methods. We performed stable isotope probing (SIP) combined with metagenomics on topsoil litter amended with WH15EPS and coupled solid culture-EPS amended medium with metagenomics.

(Continued on next page)
\end{abstract}

\footnotetext{
* Correspondence: binbinliu@sjziam.ac.cn; e.kuramae@nioo.knaw.nl

${ }^{3}$ Center for Agricultural Resources Research, Institute of Genetics and

Developmental Biology, Chinese Academy of Sciences, Shijiazhuang 050021,

Hebei, China

${ }^{1}$ Netherlands Institute of Ecology (NIOO-KNAW), Department of Microbial Ecology, Droevendaalsesteeg 10, 6708 PB Wageningen, Netherlands

Full list of author information is available at the end of the article
}

C C The Author(s). 2020 Open Access This article is licensed under a Creative Commons Attribution 4.0 International License, which permits use, sharing, adaptation, distribution and reproduction in any medium or format, as long as you give appropriate credit to the original author(s) and the source, provide a link to the Creative Commons licence, and indicate if changes were made. The images or other third party material in this article are included in the article's Creative Commons licence, unless indicated otherwise in a credit line to the material. If material is not included in the article's Creative Commons licence and your intended use is not permitted by statutory regulation or exceeds the permitted use, you will need to obtain permission directly from the copyright holder. To view a copy of this licence, visit http://creativecommons.org/licenses/by/4.0/. The Creative Commons Public Domain Dedication waiver (http://creativecommons.org/publicdomain/zero/1.0/) applies to the data made available in this article, unless otherwise stated in a credit line to the data. 
(Continued from previous page)

Results: SIP metagenome analysis of the soil litter demonstrated that phyla Proteobacteria, Actinobacteria,

Acidobacteria, and Planctomycetes were the most abundant in WH15EPS amended and unamended treatments. The enrichment cultures in solid culture medium coupled to metagenomics demonstrated an enrichment in Proteobacteria, and the metagenome assembly of this enrichment cultures resulted in 4 metagenome-assembled genomes (MAGs) of microbes with low identity (42-86\%) to known microorganisms. Among all carbohydrate-active enzymes (CAZymes) retrieved genes, glycoside transferase (GT) was the most abundant family, either in cultureindependent or culture-based metagenome datasets. Within the glycoside hydrolases (GHs), GH13 was the most abundant family in both metagenome datasets. In the "heavy" fraction of the culture-independent metagenome SIP dataset, GH109 (a-N-acetylgalactosaminidases), GH117 (agarases), GH50 (agarases), GH32 (invertases and inulinases), GH17 (endoglucanases), and GH71 (mutanases) families were more abundant in comparison with the controls. Those GH families are affiliated to microorganism that are probably capable to degrade WH15EPS and potentially applicable for biofilm deconstruction. Subsequent in culture-based metagenome, the assembled 4 MAGs (unclassified Proteobacteria) also contained GH families of interest, involving mannosidases, lysozymes, galactosidases, and chitinases.

Conclusions: We demonstrated that functional diversity induced by the presence of WH15EPS in both cultureindependent and culture-dependent approaches was enriched in GHs, such as amylases and endoglucanases that could be applied in chemical, pharmaceutical, and food industrial sectors. Furthermore, WH15EPS may be used for the investigation and isolation of yet unknown taxa, such as unclassified Proteobacteria and Planctomycetes, increasing the number of current cultured bacterial representatives with potential biotechnological traits.

\section{Background}

Metagenomics approach allows the access to a microbial genetic pool that is not reachable through classical microbial cultivation techniques. Therefore, the cultivation-independent methods have long been used as a tool for the exploration and discovery of biotechnological compounds produced by microbes in natural environments, in particular the detection of potential enzymes and other products of economic significance [1]. Culture-independent approaches allowed the clarification of potential microbial roles; however, culturebased studies are still needed for the comprehension of microbial characteristics and phenotypes [2]. The use of metagenomics has boosted industrial production systems and enzyme bioprospecting [3], particularly in animal guts [4], although other types of ecosystems, such as forest litter, remain underexplored.

Glycoside hydrolases (GHs) are among the industrially important enzymes that are extensively searched through metagenomics, as they are extremely desired and important in food and other industrial sectors [4-7]. Those enzymes are employed for brewing, baking, production of syrups, food processing, texture, flavoring, as well as the production of dairy and fermented foods [8]. GHs are also necessary for the production of biofuels, by converting cellulose and lignocellulosic biomass into sugars that can be fermented by microorganisms into bioethanol [9].

An alternative application of GHs is the degradation of polysaccharides for the removal of biofilms.
Exopolysaccharides are the main and most studied components of extracellular polymeric substances (EPS), biopolymers synthesized by a wide range of strains of microorganisms [10]. EPS are the constituents that preserve the tridimensional structure of biofilms, maintaining internal cohesion and promoting adhesion to surfaces [11]. The elimination of biofilms is important for human health in general, because those structures are implicated in several diseases, causing problems for instance in hospitals and in food processing industries [2]. Furthermore, enzymatic removal of biofilms is superior to the use of conventional cleaning agents, which are not eco-friendly, producing toxic residues, and erosion of equipment [2]. Enzymes are an environmentally friendly alternative due to their biodegradable nature [12]. EPS and biofilms are complex, requiring a wide range of enzymes for a complete degradation [11]; however, enzymes such as lysozyme, amylases, dispersin $\mathrm{B}$, and alginate lyase are already used for biofilm removal or inhibition in food and pharmaceutical industries [2]. More than 50\% of the current industrial enzymes are produced by microorganisms, such as strains of Bacillus and Aspergillus, while around $15 \%$ are derived from plants [12]. Furthermore, microbial enzymes with potential applications were obtained from habitats such as hydrothermal vents [13], arctic tundra [14], cow rumen [15], and termite guts [16].

The main goal of our study was to use a microbial EPS to target microbes and functions involved in EPS degradation in microcosm experiment with temperate forest 
litter and in culture medium. Plant litter is mostly composed of recalcitrant biopolymers, which are sources of carbon, energy, and nutrients for microbial communities living in litters layers [17]. Cellulose, hemicellulose, and pectin are the major components of plant cell walls. Cellulose is the most abundant plant cell wall component $(40-50 \%$ of the dry weight), composed of $\beta(1 \rightarrow 4)$ linear chains of D-glucose residues. Hemicelluloses $(20-30 \%$ of plant dry weight) are mostly composed of xylan, xyloglucan, $\beta$-glucan, and mannan as well as other oligosaccharides. Pectins $(10-30 \%$ of plant dry weight) contain homogalacturonan, xylogalacturonan, and rhamnogalacturonan [18]. Due to their complexity, the breakdown of plant cell wall components requires a wide range of enzymes, produced by the microorganisms during litter decomposition process [19]. Therefore, it is an interesting environment for the retrieval of complex polysaccharide-degrading enzymes. On the other hand, the microbial community in forest ecosystems is dominated by Acidobacteria [20], which phylum members are linked to carbon degradation [21]. Acidobacteria isolates belonging to Granulicella sp. from forest litter are described to produce large amounts of EPS [19]. The genus Granulicella is not a human pathogen [22], and the unique composition of its EPS is interesting for the retrieval of a wide range of glycoside hydrolase genes that could be applied in the industry for several processes [23]. The EPS of the Acidobacteria Granulicella sp. strain WH15 (WH15EPS) has a more complex composition than most commercially available microbial polymers. It is composed of 7 monosaccharides (mannose, glucose, galactose, xylose, rhamnose, glucuronic, and galacturonic acids) [23], while other known EPS are composed of maximum 4 different monosaccharides [24]. The degradation of WH15EPS would require a broader range of enzymes than other EPS; therefore, the application of WH15EPS to topsoil-litter samples would promote the enrichment of a wider range of GHs. The use of EPS as a carbon source by active microorganisms can be investigated with stable isotope probing (SIP). SIP is a robust technique that evaluates the incorporation of compounds labeled with heavy isotopes, for instance ${ }^{13} \mathrm{C},{ }^{18} \mathrm{O}$, and ${ }^{15} \mathrm{~N}$, into the cell components of microorganisms metabolizing a specific substrate [25]. Hence, SIP identifies the active microorganisms involved in the metabolism of a specific labeled compound. It has been successfully applied for the study of microorganisms incorporating several compounds, such as methanol, phenol [26, 27], and others [28].

The aim of this study was to identify potential GH microbial producers and $\mathrm{GH}$ genes with biotechnological potential, using EPS of Acidobacteria Granulicella sp. strain WH15 (WH15EPS) as an enrichment factor, in cultivation-independent and cultivation-dependent methods. We performed stable isotope probing (SIP) combined with metagenomics on topsoil litter amended with WH15EPS and coupled solid culture-EPS amended medium with metagenomics.

\section{Results \\ Overview of the metagenome data SIP metagenome}

After quality control filtering, a total of 18,762,958 reads were maintained for further analysis, with an average of $1,563,580$ reads per sample. A total of $1,209,745$ ORFs were predicted for functional annotation, and approximately $50 \%$ of these ORFs were classified using KEGG and COG databases. The sequencing statistics are in Table 1.

\section{Community composition SIP metagenome based on SSU rRNA and ORF classification}

Taxonomic annotation based on SSU rRNA annotation demonstrated that bacteria, fungi, and archaea accounted for approximately $84 \%, 4 \%$, and $2 \%$ of the sequences, respectively. At phylum level, 17 bacterial groups, 5 fungal groups, and 3 archaeal groups were observed in all the samples. The most abundant groups at phylum level belonged to domain Bacteria (Additional file 1: Supplementary Figure S1a). Proteobacteria was the most abundant phylum in all treatments $(26.4-28 \%$ of the sequences), followed by Actinobacteria (14.5-17.5\% of the sequences). In both unamended and ${ }^{12} \mathrm{C}$-EPSamended control treatments, Acidobacteria was the third most abundant group (14.5-15.8\% of the sequences), while in the "heavy" fraction samples, Planctomycetes was the third most abundant phylum (16.45\% of the sequences) (Additional file 1: Supplementary Figure S1a). At genus level, we observed 167 groups in all samples, of which 110 were unclassified groups. "Unclassified Bacteria" was the most abundant group in the unamended control (3.5\% of the sequences), while "unclassified Acidobacteriaceae" (2.6\% of the sequences) was the most abundant in the ${ }^{12} \mathrm{C}$-EPS-amended control (Fig. 1a). In labeled samples, the predominant group was "unclassified Planctomycetes" (3.2\% of the sequences) (Fig. 1a). Among the 10 most abundant groups, only 2 classified genera were observed: Acidothermus (1.8-2.9\% of the sequences) and Singulisphaera $(0.2-2.6 \%$ of the sequences) (Fig. 1a). Similarly, the taxonomic composition of the ORF-based analysis was dominated by domain Bacteria, with an average of $82 \%$ of the ORFs belonging to bacteria and approximately $18 \%$ of the ORFs originating from unclassified organisms, in all the samples (Additional file 1: Supplementary Figure S1b). At phylum level, we observed, in total, 103 bacterial groups, 6 fungal groups, and 11 archaeal groups in all the samples. Acidobacteria (20.1-25.3\% of the 
Table 1 SIP shotgun metagenomics sequencing statistics for each treatment. Average from 4 replicates

\begin{tabular}{llll}
\hline Statistics & Unamended Control & EPS amended & "Heavy" fraction \\
\hline Number of reads & $1,590,046$ & $1,591,447$ & $1,509,247$ \\
Number of contigs & $82,370.25$ & $78,474.5$ & $92,521.75$ \\
Longest contig (bp) & 2,651 & $3,645.25$ & $9,414.75$ \\
N50 & 466 & 473 & 542 \\
Mapping (\%) & 18.4 & 19.6 & 32.9 \\
Number of ORFs & 96,141 & 91,272 & $115,023.3$ \\
\hline
\end{tabular}

Unamended Control incubation without WH15EPS, EPS amended incubation containing ${ }^{12} \mathrm{C}-\mathrm{WH} 15 \mathrm{EPS}$, "Heavy" fraction, "heavy" fraction of incubations containing ${ }^{13} \mathrm{C}$-WH15EPS

sequences) was the most abundant phylum in unamended and ${ }^{12} \mathrm{C}$-EPS-amended control samples, while Actinobacteria (26\% of the sequences) was the predominant group in "heavy" fraction samples (Additional file 1: Supplementary Figure S1b). At genus level, we found 1541 groups, of which 667 were unclassified. The top three most abundant groups in both control treatments were "unclassified microorganisms" (17.3-19.4\% of the ORFs), "unclassified Bacteria" (12.7-16\% of the ORFs), and "unclassified Acidobacteriaceae" (9.3-11.5\%), while the predominant groups in "heavy" fraction samples were "unclassified microorganisms" (16.1\% of the ORFs), "unclassified Bacteria" (18.9\% of the ORFs), and "unclassified Planctomycetes" (9\% of the ORFs) (Fig. 1b).

PERMANOVA ( $p$ values $<0.001$ ) showed that, for both SSU rRNA data and ORF-based analysis, the microbial communities were different between treatments, with both control treatments closer to each other, and "heavy" fraction samples separated from both control treatments in PCoA graphs (Fig. 2). For SSU rRNA communities, the first two axes of PCoA explained $43.3 \%$ of the variation, while for ORF based data, $90.6 \%$ of the variation was explained. RDA analysis $(p=0.002)$ for both datasets showed that mainly groups of Planctomycetes, such as "unclassified Planctomycetes", "unclassified Planctomycetales," "unclassified Planctomycetia" and Singulisphaera, were driving the dispersion of the microbial communities between "heavy" fraction and both control treatments (Additional file 1: Supplementary Figure S2), consistently with the higher abundance of Planctomycetes in labeled samples. Alpha diversity indices showed that richness and diversity indices were lower for "heavy" fraction samples in comparison with both controls (Additional file 1: Supplementary Figure S3), supported by ANOVA test ( $p$ value $<0.05$ ).

\section{Functional profile of SIP metagenome}

KEGG, COG, and CAZy databases were employed for functional gene annotation to explore the functional characteristics of the microbial communities. Approximately $60 \%$ of the ORFs were assigned to COGs, matching in total to 20,644 COGs. The most abundant COG categories in all the samples were "R-general function prediction" (10.8-11.6\% of the ORFs) (Additional file 1: a)

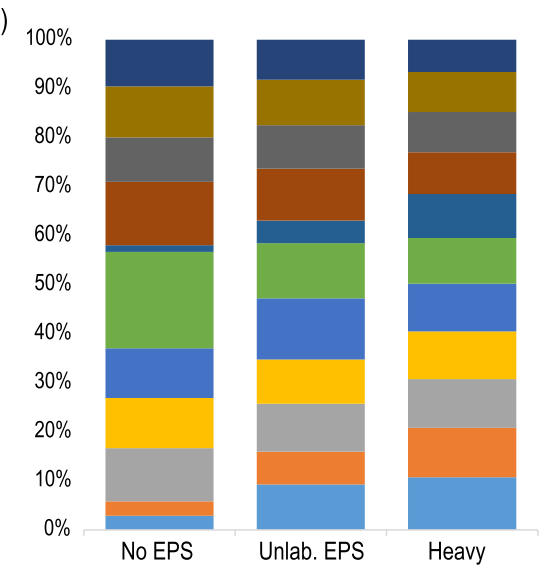

b)

-Unc. Eukaryota
-Unc. Acidobacteriales
-Unc. Alphaproteobacteria
-Unc. Gammaproteobacteria
- Singulisphaera
-Unc. bacteria
-Unc. Acidobacteriaceae
- Acidothermus
-Unc. Acidimicrobiia
-Unc. Armatimonadales
-Unc. Planctomycetes

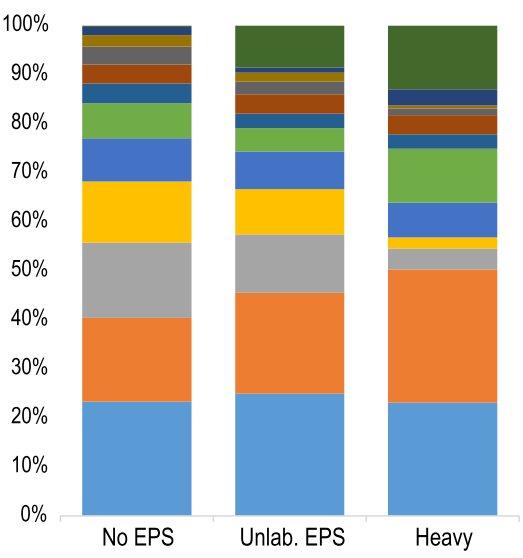

- Unc. Planctomycetes - Unc. Streptosporangiales - Uncl. Acidobacteriales - Unc. Rhizobiales - Unc. Gammaproteobacteria - Unc. Alphaproteobacteria - Unc. Actinobacteria - Unc. Proteobacteria - Unc. Acidobacteria - Unc. Acidobacteriaceae - Unc. bacteria - Unc. microorganisms

Fig. 1 Taxonomic composition and relative abundance of microbial groups at genus level in SIP metagenome treatments based on a SSU rRNA gene taxonomic classification and $\mathbf{b}$ ORF taxonomic classification. Only the ten most abundant groups for each treatment are displayed. Average abundances of 4 replicates. Unc.: unclassified. No EPS: incubation without WH15EPS. Unlab.: EPS-incubation containing ${ }^{12} \mathrm{C}-$ WH15EPS. Heavy: "heavy fraction" of incubations containing ${ }^{13} \mathrm{C}-$ WH15EPS 

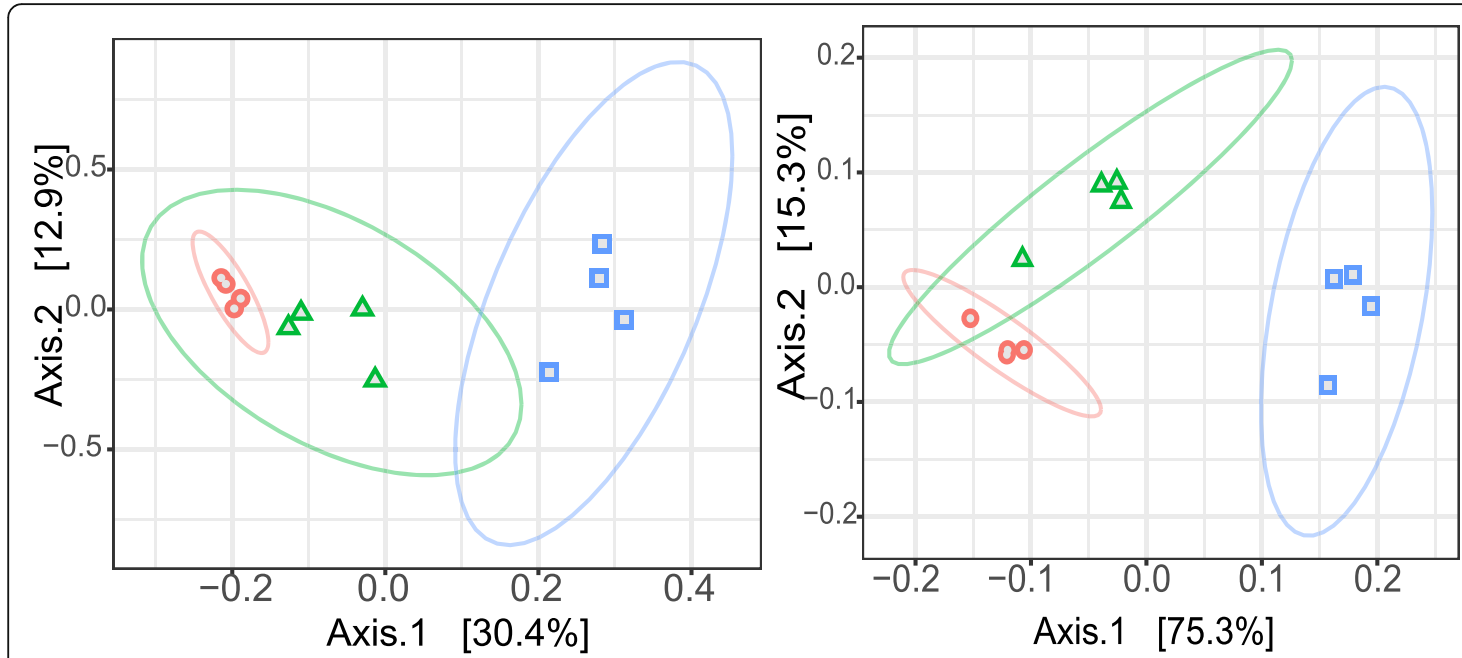

Group -. No EPS $\triangle$ Unlab. EPS - Heavy

Fig. 2 Principal Coordinate Analysis (PCOA) clustering of normalized and Hellinger-transformed SIP metagenome sequencing data based on BrayCurtis distances of a SSU rRNA gene taxonomic classification and $\mathbf{b}$ ORF taxonomic classification. No EPS: incubation without WH15EPS. Unlab.: EPS-incubation containing ${ }^{12} \mathrm{C}-\mathrm{WH} 15$ EPS. Heavy: "heavy fraction" of incubations containing ${ }^{13} \mathrm{C}-\mathrm{WH} 15 \mathrm{EPS}$

Supplementary Figure S4a). Boruta feature selection "random forest" analysis $(p<0.05)$ was used to identify feature annotations that segregated significantly between treatments. A total of 32 COGs were selected by Boruta algorithm. Thirteen among the identified COGs were more abundant in the unamended control samples, while 19 were more abundant in the labeled samples (Fig. 3a). However, most of the features identified by the analysis belonged to the category unknown function. Some of the unknown COGs abundant in the labeled treatment, though, were associated mostly to phyla Planctomycetes and Acidobacteria, according to eggNOG database v 4.5 (Additional file 1: Supplementary Table S1).

KEGG analysis demonstrated that about $50 \%$ of the ORFs were assigned to 7,343 KEGG functional orthologs. The 17 most abundant KEGGs in all samples were assigned to three categories: signaling and cellular processes (8 KEGGs-0.16\% of the total ORFs), genetic information and processing (6 KEGGs- $0.14 \%$ of the total ORFs), and metabolism (3-0.21\% of the total ORFs) (Additional file 1: Supplementary Figure S4b). Boruta feature selection identified 40 KEGGs that influenced the dispersion of the samples, of which 26 were more abundant in the labeled treatment and 14 were more abundant in the unamended control (Fig. 3b). Among the KEGGs more abundant in the labeled treatment, 13 could be assigned to KEGG pathways, mostly related to "metabolic pathways" and "microbial metabolism in diverse environments" (Additional file 1: Supplementary Table S2). Within the KEGGs more abundant in the unamended control treatment, 8 could be assigned to KEGG pathways, the majority related to "metabolic pathways" (Fig. 3b, Additional file 1: Supplementary Table S2).

Annotation using dbCAN database showed that families GT41 (8.4-11\% of the CAZYmes), AA3 (4.4-5\%), GT4 (3.4-4.7\%), GT2 (4.1-4.3\%), and CE10 (3.5-4.2\%) were among the most predominant in all the treatments (Additional file 1: Supplementary Figure S4c). Boruta feature selection identified 27 CAZY families affecting the dispersion of the sample treatments (Fig. $3 \mathrm{c}$ ), the vast majority belonging to the category glycoside hydrolase $(\mathrm{GH})$. Among the selected families, 15 were more abundant in the labeled treatment, and 12 were more abundant in the unamended control. The categories abundant in the labeled treatment involved xylan and fructan modules, xylanases, mannosyltransferases, and agarases, while the categories abundant in the unamended controls were mostly $\alpha$ and $\beta$ galactosidases and glucosidases (Additional file 1: Supplementary Table S3). PERMANOVA ( $p$ values < 0.001) demonstrated that for KEGG, COG, and dbCAN data, the functional gene compositions were different between treatments, similarly to taxonomic analysis, with control treatments grouping together and separated from "heavy" fraction samples (Additional file 1: Supplementary Figure S5).

\section{Cultivated microbes metagenome Overview of the metagenomics data}

A total of $422,735,048$ reads were obtained after sequence quality filtering, with an average of $80 \%$ of the ORFs classified with KEGG and COG databases. The sequencing statistics are described in Table 2. 


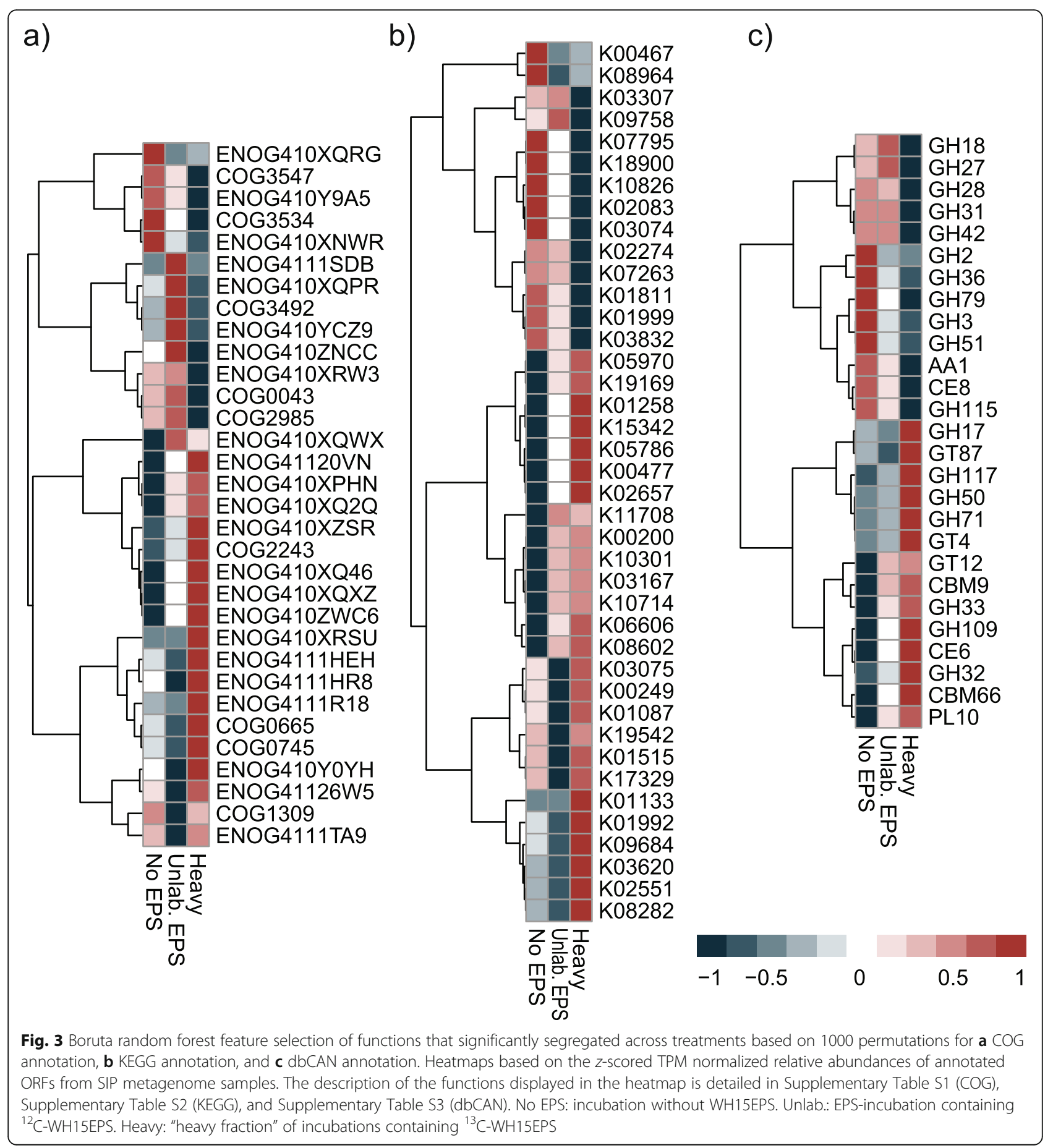

\section{Community composition of cultivated microbes} metagenome based on SSU rRNA and ORF classification Analysis of the taxonomic composition based on SSU rRNA showed an average of $73 \%$ of the sequences belonged to domain Bacteria, 20\% to kingdom Fungi, and $7 \%$ were derived from other Eukaryotes (Additional file 1: Supplementary Figure S6a). At phylum level, 17 bacterial groups, 7 fungal groups, and 14 eukaryotic groups were identified. The most abundant group was the bacterial phylum Proteobacteria, with $\sim 47.9 \%$ of the sequences, followed by fungal phylum Ascomycota, with $\sim 14.5 \%$ of the sequences (Additional file 1: Supplementary Figure S6b). At genus level, 450 groups in total were observed, with the most abundant groups being bacterial groups. The predominant groups were "unclassified $\mathrm{Bac}$ teria" ( $2.2 \%$ of the sequences) and Dyella $(\sim 1.5 \%$ of 
Table 2 Cultivated shotgun metagenome sequencing statistics for each plate. Average from 2 replicates per plate

\begin{tabular}{lllll}
\hline Statistics & Plate 1 & Plate 2 & Plate 3 & Plate 4 \\
\hline Number of reads & $49,148,370$ & $54,247,258.5$ & $58,397,852$ & $49,574,043.5$ \\
Assembled reads & $1.47 \mathrm{E}+10$ & $1.6224 \mathrm{E}+10$ & $1.75 \mathrm{E}+10$ & $1.4796 \mathrm{E}+10$ \\
Number of contigs & $67,980,868$ & $76,125,070.5$ & $82,202,170$ & $68,767,888$ \\
Number of predicted genes & 159,832 & 254,727 & 535,677 & 479,683 \\
KEGG (\% classified ORFs) & 66.2 & 67.2 & 65.2 & 63.9 \\
COG (\% classified ORFs) & 94.6 & 94.5 & 94.1 & 4.7 \\
CAZYmes (\%) & 4.5 & 4.7 & 4.7 & 4.5 \\
GC content (\%) & 59.6 & 60.3 & 59.0 & 58.2 \\
\hline
\end{tabular}

the sequences) (Fig. 4a). Silvimonas and Burkholderia were also among the top 10 most abundant genera ( 1.4 and $1.3 \%$ of the sequences, respectively). Similarly, for the ORF based data, the most abundant groups at genus level belonged to domain Bacteria, revealing the presence of 1930 groups at genus level. "Unclassified microbes" was the most abundant group, followed by genera Caballeronia (15.4\% of the ORFs) and Paraburkholderia (15.1\% of the ORFs) (Fig. 4b). Other genera, such as Burkholderia, Rhodanobacter, and Dyella were also among the predominant groups $(7.8,7.1$, and $4.9 \%$ of the ORFs) (Fig. 4b).

\section{Functional profile of cultivated microbes metagenome}

The functional profile of the cultivated microbes' metagenome was explored through the annotation with KEGG, COG, and dbCAN databases. COG analysis demonstrated that approximately $20.6 \%$ of the annotated
COGs were assigned to unknown functions. Among the classified COGs, similarly to SIP metagenome, the predominant categories involved "E-amino acid transport and metabolism" ( 8.6\% of the ORFs), "G-carbohydrate transport and metabolism" ( $8.0 \%$ of the ORFs), and "C-energy production and conversion" ( 7.3\% of the ORFs) (Fig. 5a).

KEGG pathway analysis showed that around 65\% of the ORFs were assigned to 9945 KEGG orthologs. The 20 most abundant KEGGs were distributed in the categories "Genetic information processing" (1 KEGG $0.24 \%$ of the total ORFs), "Metabolism" (4 KEGGs $1.18 \%$ of the total ORFs), and "Signaling and cellular processes" (15 KEGGs - 4.54\% of the ORFs), of which 13 KEGGs were classified as transporters (Fig. 5b).

The analysis of the carbohydrate-active enzymes with dbCAN demonstrated the presence of 298 CAZyme families. Twenty-three families were predominant, which

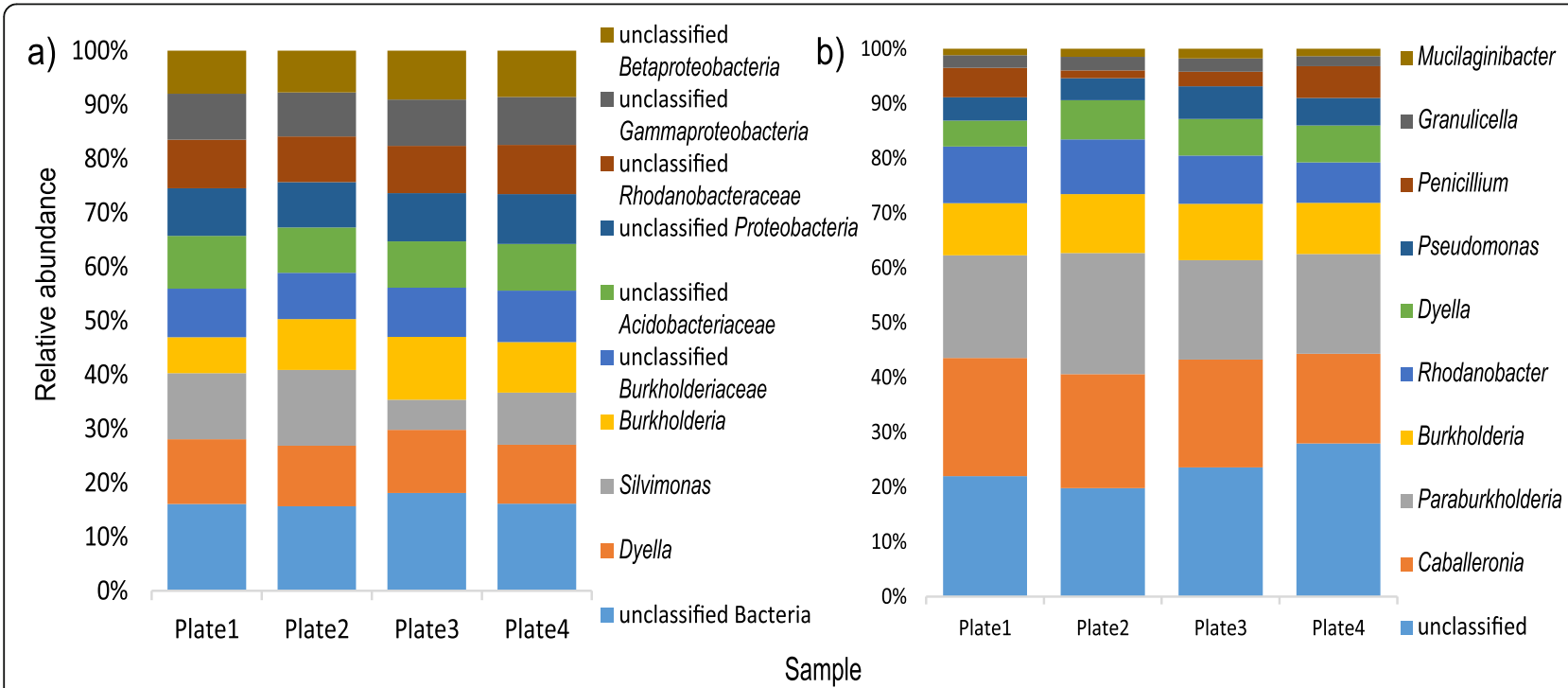

Fig. 4 Taxonomic composition and relative abundance of microbial groups at genus level in samples from the metagenome shotgun of cultivated microorganism based on a SSU rRNA gene taxonomic classification and $\mathbf{b}$ ORF taxonomic classification. Only the ten most abundant groups are displayed. Average from 2 replicates per plate of culture medium 


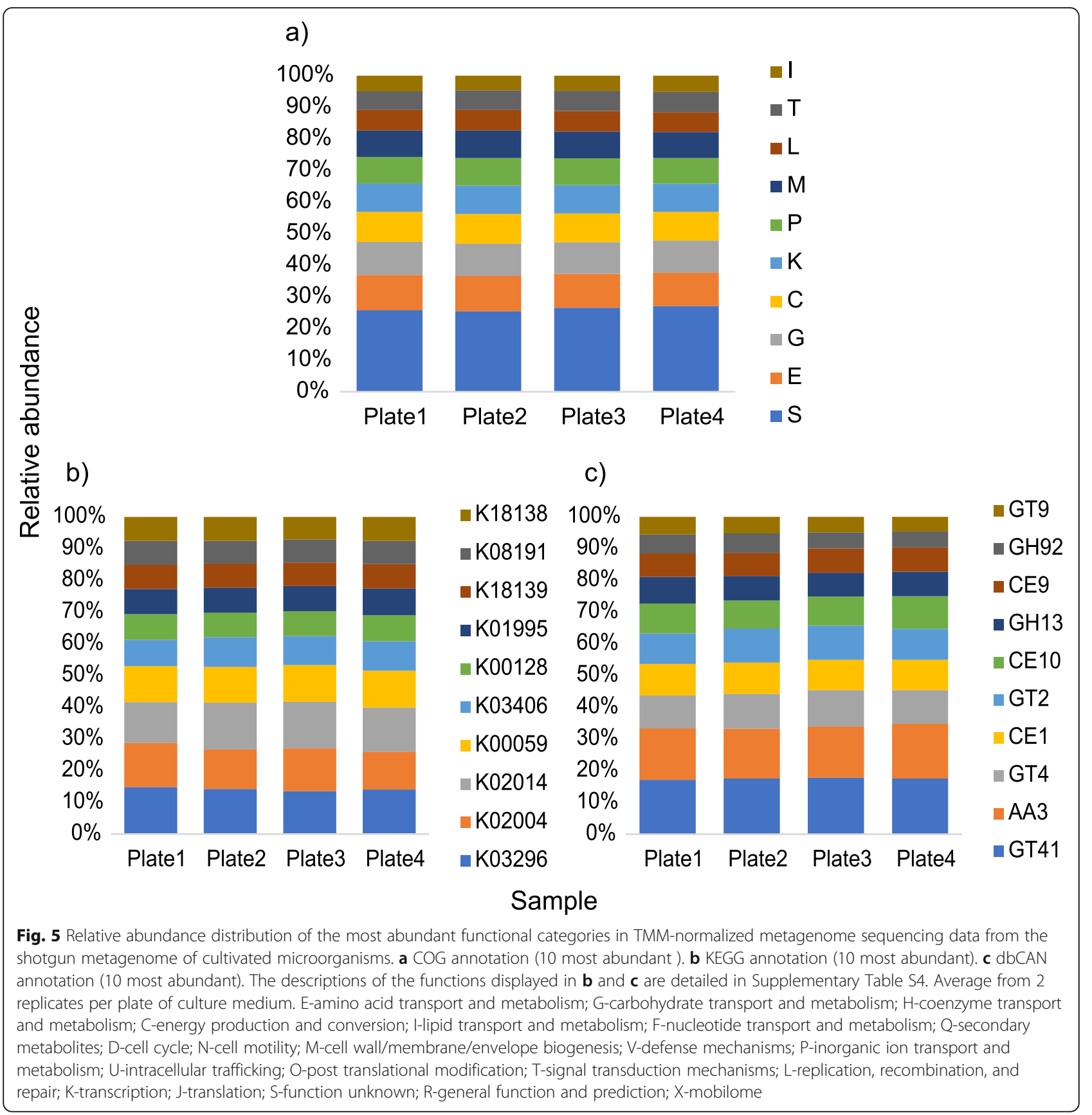

abundance was above $1 \%$. Within the most abundant families, we observed 2 AA families (7.75\% of the CAZymes), 1 CBM family, $4 \mathrm{CE}$ families, $10 \mathrm{GH}$ families, and $6 \mathrm{GT}$ families (Fig. 5c). Those CAZyme families comprise mostly enzymes with cellulolytic (alpha-glucosidases, alphafucosidases), hemicellulolytic (alpha-rhamnosidases, alphaxylosidases, alpha-mannosidases, beta-galactosidases), and cell wall metabolism activities ( $\mathrm{N}$-acetylglucosaminyltransferases, alpha- $\mathrm{N}$-acetylgalactosaminidases, and peptidoglycan lyases) (Additional file 1: Supplementary Table S4). The most abundant family was GT41 (Fig. 5c), which encompasses UDP-GlcNAc: peptide $\beta$-N-acetylglucosaminyltrans ferases and UDP-Glc: peptide N- $\beta$-glucosyltransferases, enzymes involved in protein glycosilation. Among the GH families, the most abundant was GH13.

Among all $127 \mathrm{GH}$ families found in both metagenome datasets, 114 families were observed in both datasets, while 5 families were exclusive from the SIP dataset (GH112, GH48, GH52, GH86, GH98) and 8 were exclusive from the cultivated microbes dataset (GH111, 
GH131, GH132, GH134, GH45, GH7, GH80, GH85) (Additional file 1: Supplementary Figure S7).

\section{Taxonomy of the enriched glycoside hydrolase families}

Taxonomic analysis of the most abundant GH family in both metagenome datasets, GH13, demonstrated that the majority of the sequences of GH13 in the cultivated microbes dataset belonged to phyla Proteobacteria (66.8\% of the GH sequences) and Acidobacteria (21.8\% of the GH sequences), while in the SIP dataset the most abundant phyla for GH13 were Actinobacteria (20.4$45.7 \%$ of the GH sequences), Acidobacteria (4-24.7\% of the sequences), and other phyla (27-34\% of the GH sequences) (Table 3).

Within GH families that were more abundant in the SIP "heavy" fraction (Fig. 3c), sequences of GH109 belonged mainly to Acidobacteria (45\% of the GH sequences), other phyla (31-42\% of the GH sequences), and Planctomycetes (2-29\% of the GH sequences). GH117 family sequences belonged predominantly to Actinobacteria (17-33\% of the sequences), Acidobacteria (0-33\% of the GH sequences), and other phyla (33-64\% of the GH sequences). Family GH50 sequences belonged mainly to Proteobacteria (8-100\% of the GH sequences) and other phyla (0-92\% of the GH sequences). GH 32 sequences were affiliated mainly to Acidobacteria (11$44 \%$ of the GH sequences) and other phyla (44-79\% of the GH sequences). GH17 sequences belonged to phylum Proteobacteria (44-75\% of the GH sequences) and other phyla (25-57\% of the GH sequences). GH71 sequences were affiliated to phyla Actinobacteria (35$100 \%$ of the GH sequences), Proteobacteria (0-25\% of the sequences), Acidobacteria (0-25\% of the sequences), and other phyla ( $0-43 \%$ of the sequences).

\section{Metagenome-assembled genomes (MAGs) assembled from the cultivated microbes metagenome}

The binning process using contigs longer than $5 \mathrm{~kb}$ generated, after curation and quality filtering, $4 \mathrm{draft}$ genomes. The genome length ranged from 3.0 to 6.3 $\mathrm{Mb}$, and the GC content ranged from 57 to $62 \%$. All MAGs belonged to phylum Proteobacteria. None of the MAGs was classified to genus level; however, the genomes were closer to genera Paraburkholderia (MAG1) and Amantichitinum (MAG2 and MAG4). MAG3 closest classification was to family Rhodanobacteraceae.

Table 3 Taxonomy associated to sequences of glycoside hydrolases belonging to GH13 family (most abundant) and the enriched $\mathrm{GH}$ families in heavy fraction samples from SIP metagenome

\begin{tabular}{|c|c|c|c|c|c|c|}
\hline GH families & Sample & Proteobacteria & Acidobacteria & Actinobacteria & Planctomycetes & Others \\
\hline GH13 & Cultivated & $66.8(2605)$ & $21.8(827)$ & $0.9(36)$ & $0.09(1)$ & $10.4(51.4)$ \\
\hline \multirow[t]{3}{*}{ GH13_SIP } & Control & $20.1(128)$ & $23.4(148)$ & $25.6(162)$ & $0.1(4)$ & 31 (196) \\
\hline & EPS & $17.9(89)$ & $24.7(125)$ & $20.4(95)$ & $2.9(13)$ & $34(172)$ \\
\hline & Labeled & $17.2(127)$ & $4(30)$ & 45.7 (333) & $5.7(42)$ & $27(201)$ \\
\hline \multirow[t]{3}{*}{ GH109 } & Control & $11(22)$ & $45(92)$ & $12(26)$ & $2(4)$ & $31(68)$ \\
\hline & EPS & $7(19)$ & $26(76)$ & $7(18)$ & $21(60.8)$ & $40(111.6)$ \\
\hline & Labeled & $7(34)$ & $9(48)$ & $13(62)$ & $29(150)$ & $42(217)$ \\
\hline \multirow[t]{3}{*}{ GH117 } & Control & $0(0)$ & $33(1)$ & $33(1)$ & $0(0)$ & $33(1)$ \\
\hline & EPS & $0(0)$ & $0(0)$ & $17(1)$ & $0(0)$ & $38(5)$ \\
\hline & Labeled & $9(1)$ & $0(0)$ & $27(3)$ & $0(0)$ & $64(7)$ \\
\hline \multirow[t]{3}{*}{ GH50 } & Control & $100(3)$ & $0(0)$ & $0(0)$ & $0(0)$ & $0(0)$ \\
\hline & EPS & $100(2)$ & $0(0)$ & $0(0)$ & $0(0)$ & $0(0)$ \\
\hline & Labeled & $8(2)$ & $0(0)$ & $0(0)$ & $0(0)$ & $92(24)$ \\
\hline \multirow[t]{3}{*}{ GH32 } & Control & $0(0)$ & $44(4)$ & $11(1)$ & $0(0)$ & $44(4)$ \\
\hline & EPS & $0(0)$ & $11(2)$ & $5(1)$ & $5(1)$ & $79(15)$ \\
\hline & Labeled & $6(2)$ & $14(5)$ & $3(1)$ & $9(3)$ & $69(24)$ \\
\hline \multirow[t]{3}{*}{ GH17 } & Control & $75(9)$ & $0(0)$ & $0(0)$ & $0(0)$ & $25(3)$ \\
\hline & EPS & $43(3)$ & $0(0)$ & $0(0)$ & $0(0)$ & $57(4)$ \\
\hline & Labeled & $44(8)$ & $0(0)$ & $0(0)$ & $0(0)$ & $56(10)$ \\
\hline \multirow[t]{3}{*}{ GH71 } & Control & $0(0)$ & $0(0)$ & $100(1)$ & $0(0)$ & $0(0)$ \\
\hline & EPS & $0(0)$ & $25(1)$ & $50(2)$ & $0(0)$ & $25(1)$ \\
\hline & Labeled & $22(5)$ & $0(0)$ & $35(8)$ & $0(0)$ & $43(10)$ \\
\hline
\end{tabular}


Table 4 Genome characteristics for the 4 metagenome-assembled genomes (MAGs) obtained in this study

\begin{tabular}{|c|c|c|c|c|}
\hline Genome & MAG1 & MAG2 & MAG3 & MAG4 \\
\hline $\begin{array}{l}\text { Taxonomy } \\
\text { (closest hit) }\end{array}$ & $\begin{array}{l}\text { Burkholderiaceae 95\% } \\
\text { (Paraburkholderia 86\%) }\end{array}$ & $\begin{array}{l}\text { Neisseriaceae } 42 \% \\
\text { (Amantichitinum: 42\%) }\end{array}$ & $\begin{array}{l}\text { Rhodanobacteraceae } \\
77 \%\end{array}$ & $\begin{array}{l}\text { Neisseriaceae: } 42 \% \\
\text { (Amantichitinum 42\%) }\end{array}$ \\
\hline Length (Mb) & 6.3 & 3.0 & 4.8 & 3.7 \\
\hline Contigs & 1997 & 997 & 80 & 1482 \\
\hline Completeness (\%) & 83.2 & 79.6 & 99.7 & 87.5 \\
\hline Contamination (\%) & 4.76 & 3.92 & 2.44 & 5 \\
\hline GC (\%) & 62 & 57 & 59 & 57 \\
\hline Number of predicted genes & 7126 & 3580 & 4280 & 4552 \\
\hline \multicolumn{5}{|l|}{ Hits to protein database } \\
\hline KEGG \% & 90.1 & 96.8 & 79.2 & 93.8 \\
\hline COG \% & 85.3 & 85.1 & 80.2 & 84 \\
\hline DBcan $n(\%)$ & $279(3.9)$ & $141(3.9)$ & $210(4.9)$ & $180(4.0)$ \\
\hline
\end{tabular}

The characteristics of the genomes are described in Table 4. The coverage of the genomes is described in Additional file 1: Supplementary Table S5.

Approximately, $83.7 \%$ of the ORFs predicted for the MAGs could be assigned to COGs. The analysis showed that most of the COG assigned ORFs fell on the category "S-function unknown" (16.4-18.4\% of the ORFs). Among the classified COGs, however, the most abundant categories were "K-transcription" (5.9-9\% of the ORFs), "E-amino acid metabolism" $(4.8-8.1 \%$ of the ORFs), "G-carbohydrate metabolism" (3.32-7.2\%), "Cenergy production" (4.2-5.9\%), "P-inorganic ion metabolism" (4.65-6.3\%), and "M-cell wall/membrane biogenesis" (5.2-5.9\%) (Fig. 6a).

KEGG pathway analysis demonstrated that around $90 \%$ of the predicted ORFs could be assigned to KEGG orthologs. The majority of the most abundant KEGG orthologs in all the MAGs were related to several types of transporter functions (Fig. $6 \mathrm{~b}$ and Additional file 1: Supplementary Table S6). In order to evaluate the features of the MAGs that could be involved in the uptake of the WH15EPS sugar units, we decided to look deeper into the transporters. Twenty-four of the KEGG orthologs observed in MAG1 genome were associated to the transport of several sugars, such as sorbitol, ribose, arabinose, xylose, fructose, rhamnose, glucose, mannose, and multiple sugars (Additional file 1: Supplementary Table S7). Among the KEGG orthologs observed in MAG 2 genome, 62 were related to sugar transport, such as maltose, raffinose, lactose, glucosides, cellobiose, xylose, fructose, rhamnose, glucose, mannose, and multiple sugars (Additional file 1: Supplementary Table S8). MAG3 did not exhibit sugar specific transporters within the 60 KEGGs related to transport function; however, we observed some general type transporters (Additional file 1: Supplementary Table S9). In MAG4, 61 KEGG orthologs related to sugar transport were observed, such as maltose, raffinose, lactose, sorbitol, cellobiose, arabinose, xylose, fructose, rhamnose, glucose, mannose, and multiple sugars (Additional file 1: Supplementary Table S10). We also performed the analysis of the CAZYmes with dbCAN database, in order to find enzymes that could be in associated the breakdown of the WH15EPS. MAG1 possessed 279 CAZymes distributed in 90 families, of which the most abundant were CE1, GT4, GT42, CE10, and AA3 (Fig. 6c). The seventy-six glycoside hydrolases observed were distributed in 43 families, including a wide range of activities, such as endo and exo-mannosidases, alpha- and beta-glucosidases and galactosidases, xylosidases, fucosidases, and rhamnosidases (Additional file 1: Supplementary Table S11). MAG2 possessed 141 CAZymes distributed in 65 families, and GT41, GT2, and CE1 were the most abundant families (Fig. 6c). A total of 51 glycoside hydrolases from 30 families were observed, with activities such as alpha- and beta-glucosidases, beta-galactosidases, mannanases and mannosidases, xylanases, and polygalacturonases (Additional file 1: Supplementary Table S11). In MAG3, 210 cazymes distributed in 81 families were observed, and GT41, GT2, CE1, and CE10 were the most abundant (Fig. 6c). Sixty-four glycosil-hidrolases distributed in 37 families were detected. The activities included alphaand beta-galactosidases, alpha-glucosidases, mannosidases, mannanases, rhamnosidases, arabinosidades, chitinases, and trehalases (Additional file 1: Supplementary Table S11). The genome of MAG4 displayed 180 CAZymes distributed in 73 families, of which the most abundant were CE1, GT2, and GT41 (Fig. 6c). The 64 glycoside hidrolases were spread among 34 families, including activities such as chitinases, arabinofuranosidases, alpha- and beta-glycosidases, mannosidases, cellulases, xylanases, and polygaracturonases (Additional file 1: Supplementary Table S11). The distribution of most abundant CAZYmes and GH families in both 


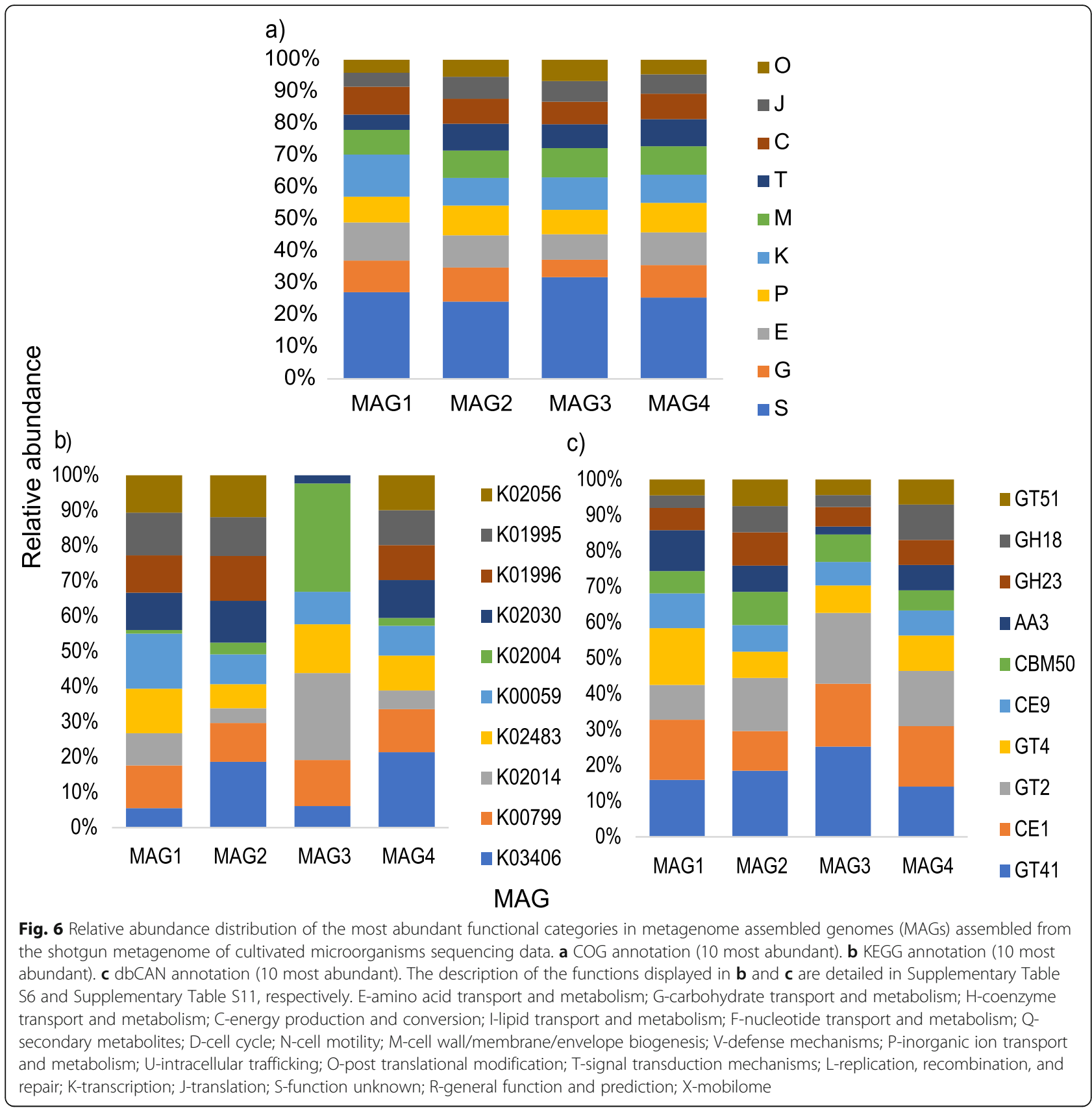

metagenomics datasets and MAGs is depicted in Additional file 1: Supplementary Figure S8.

\section{Discussion}

In the present study, we applied culture-independent and culture-dependent techniques to evaluate microbial diversity and functions involved in the degradation of a microbial biopolymer, WH15EPS, focusing on enzymes of biotechnological interest. First, we compared the functional potential of the environment with and without the presence of WH15EPS, evaluating the taxonomic and functional enrichment produced by the addition of the biopolymer using stable isotope probing (SIP). Second, we used metagenomics to evaluate the functional potential of the microorganisms grown in culture medium with WH15EPS as the sole carbon source.

SIP analysis demonstrated that in both 16S rRNAmetagenome dataset extracted and ORF based characterization, phyla Proteobacteria, Actinobacteria, Acidobacteria, and Planctomycetes were the most abundant in WH15EPS amended and unamended treatments. However, the addition of WH15EPS to the litter samples 
promoted an increase in the abundance of the phylum Planctomycetes, which was more evident in "heavy" fraction samples, showing that Planctomycetes also play an active part in the degradation of WH15EPS. Furthermore, at genus level in the $16 \mathrm{~S}$ rRNA based analysis, "unclassified Planctomycetes" and Singulisphaera, which belong to the same phylum, were the most abundant groups in the labeled treatment, while "unclassified Planctomycetes" was also among the most abundant in the ORF-based analysis. Proteobacteria, Actinobacteria, and Acidobacteria are widely known to be involved in carbon-degradation processes, for instance, glucose [29], xylan [30], and cellulose assimilation [31]. The glycolytic potential of phylum Planctomycetes was recently demonstrated by Ivanova et al. [32], in which genus Singulisphaera, for instance, responded significantly to pectin and xylan amendments.

The cultivation-dependent approach demonstrated, as expected, a lower taxonomic diversity, in which the widely studied Proteobacteria were among the most abundant. The discrepancy between the diversity of taxa, especially the most abundant groups, observed in cultured and uncultured-based techniques is defined as "The Great Plate Count Anomaly" [33]. The cultivability of microorganisms in laboratory depends of many factors, such as nutrients, oxygen level, temperature, $\mathrm{pH}$, and growing factors [34], limiting the total assortment of taxa that can be actually recovered in culture media. Nevertheless, adding WH15EPS as an alternative carbon source allowed us to demonstrate that several still unknown microorganisms can be grown in laboratorial conditions if unusual compounds are explored. The lower diversity in the culture media plates permitted the assembly of 4 draft genomes related to the most abundant Proteobacteria, which classification until genus level was not possible, once more demonstrating the enrichment and potential for isolation of previously unknown microbes.

In order to find potential enzymes of biotechnological interest, we investigated the diversity of CAZymes in both culture-independent and culture-dependent generated datasets, due to their importance in almost all industrial sectors, such as chemical, pharmaceutical, and food industries, as well as production of detergents, textiles, leather, paper, and bioenergy [4]. Furthermore, we also investigated the presence of enzymes that could be employed for biofilm removal.

Among all CAZymes observed, the most abundant families belonged to glycoside transferase families, such as GT41, GT2, and GT4, either in culture-based or in culture-independent datasets. GTs are known to catalyze the formation of glycosidic bonds by transferring a sugar residue from a donor to an acceptor, which could be carbohydrates, proteins, lipids, DNA, and other molecules [35]. Even though a large proportion of genes of microorganism's genomes in general encode for GTs (about 1-2\% of the total number of genes) [36], those enzymes are still not as well explored as GHs [35]. Glycosilated compounds play a wide range of roles, such as energy storage, cell integrity and signaling, among others, and the glycosilation of natural products is important in the exploration of bioactive compounds [37]. GTs are involved in the production of antibiotics, such as chloroeremomycin [38], vancomycin [39], and erythromycin D [40]; therefore, they might be of interest especially for the pharmaceutical industry.

Within glycoside hydrolases, the most abundant family in both metagenomics datasets was GH13 (from Proteobacteria), which encompasses starch and pullulan modifying enzymes, including $\alpha$-amylases, pullulanases, $\alpha-1,6$ glucosidases, branching enzymes, maltogenic amylases, neopullulanases, and cyclodextrinases [41]. Amylases are among the most important enzymes for food industry, where they can be employed for production of glucose and maltose syrups, reduction of viscosity of syrups, production of clarified fruit juices, solubilization of starch for brewing processes, and manufacture of baked products [12]. Furthermore, the application of $\alpha$-amylases for the inhibition of biofilm formation has been investigated. In the study of Fleming et al. [42], the use of amylase (from Bacillus subtilis) and cellulose (from Aspergillus niger) solutions to biofilms of $S$. aureus and $P$. aeruginosa decreased biomass significantly, increasing the effectiveness of antibiotics treatments. A similar effect was observed in the study of Craigen et al. [43], where a commercially available $\alpha$-amylase detached the aggregates produced by $S$. aureus and inhibited biofilm production.

Notwithstanding, feature selection with Boruta package revealed the differential abundance of GH families in "heavy" fraction SIP samples, originated from microorganisms that are believed to be able to degrade WH15EPS. These microorganisms belonged mainly to phyla Proteobacteria, Acidobacteria, Actinobacteria, Planctomycetes, as well as high proportion of unknown microorganisms. GH109 (Acidobacteria and Planctomycetes) contains $\alpha$-N-acetylgalactosaminidases, which might be employed in the development of universal red blood cells, through the enzymatic removal of monosaccharides from red blood cells' membranes, and improvement of blood supply in hospitals [44]. Furthermore, those enzymes can be involved in the deconstruction of WH15EPS, since it contains units of xylose, glucose, and arabinose [23]. Families GH117 (Acidobacteria and Actinobacteria) and GH50 (Proteobacteria) contain agarases, which can be used for the production of oligosaccharides with antioxidant activities for applications in food, pharmaceutical, and cosmetic industries [45]. 
Family GH32 (Acidobacteria) comprises invertases and inulinases, enzymes that can be applied in food and fermentation processes [46, 47]. GH17 (Proteobacteria) is composed of endoglucanases with activity against $\beta$ glucan and laminarin, effective additives for the degradation of polysaccharides for animal feed [47]. Mutanases belonging to GH71 (Actinobacteria) family already showed activity against glucans present in dental plaque [48].

Interestingly, sixteen of the most abundant GH families in the culture-independent dataset were found to be the predominant in the culture-dependent approach, and all the GH families with higher abundances in the labeled SIP samples were also observed in the culturedependent dataset. Furthermore, the MAGs also contained GH families of interest, with variable abundances among them. MAG1 (similar to Paraburkholderia) contained 8 ORFs belonging to family GH92, which encompasses alpha-mannosidases with applications in food and pharmaceutical industries, for the production of juices, degradation of plant material, or coffee extraction [49]. In MAG2 (similar to Amantichitinum), five ORFs were classified as GH23, which contains lysozymes that can be used as polysaccharide hydrolysers for biofilm breakdown [2, 50]. MAG3 (Rhodanobacteraceae) is abundant in GH92 and GH23 but also GH2 family ORFs, which comprises several enzymes. Within the best characterized ones, there are $\beta$-galactosidases employed for the production of lactose-free milk products and other galactooligosaccharides [51]. MAG4 (similar to Amantichitinum) is rich in GH18 enzymes, involving chitinases that for instance are important agents with applications for fungal biological control and bioremediation processes [52]. It is important to recognize that, even though the MAGs possessed a low level of contamination $(<5 \%)$, they do not represent genomes of axenic cultures from isolated microorganisms. Therefore, the corresponding laboratory cultures should still be recovered in order to fully validate our MAGs.

Our study showed that, using SIP and a complex EPS (WH15EPS), we could detect the subset of the total microbial community that was capable of incorporating the biopolymer. Among those we observed members of Planctomycetes as an interesting target for biotechnological studies and heterologous expression, which could be performed also in several other genes, combining bioinformatics, gene synthesis, and enzymatic screening [53]. In addition, we demonstrated that functional diversity induced by the presence of WH15EPS in both culture-dependent and culture-independent approaches was enriched in genes coding for GHs, for instance, amylases, chitinases, agarases, and endoglucanases and that could be applied in chemical, pharmaceutical, and food industries. Furthermore, the use of WH15EPS may be employed for the investigation and isolation of yet unknown taxa, such as unclassified Proteobacteria and Planctomycetes, increasing the number of current cultured bacterial representatives.

\section{Conclusions}

We observed, in the functional diversity induced by the presence of WH15EPS in both culture-dependent and culture-independent approaches, the presence of 310 CAZyme families, from which $38.4 \%$ (119) were GH families. GHs of biotechnological interest could potentially be employed in almost all industrial sectors, such as chemical, pharmaceutical, and food industries, as well as production of detergents, textiles, leather, paper, and bioenergy. Furthermore, we also observed the presence of enzymes that could be employed for biofilm removal. Even though the potential enzymes might belong to slow growing microorganisms in laboratorial conditions, such as Acidobacteria, Planctomycetes, and Verrucomicrobia, sequences can still be targeted for further heterologous expression and characterization. In addition, the culturebased metagenomics dataset allowed the assembly of 4 metagenome-assembled genomes (MAGs) that potentially belong to unclassified Proteobacteria. We showed that WH15EPS may be employed for the isolation of known and unknown microbes, as well as the targeting of sequences of a wide range of CAZyme families.

\section{Material and methods \\ Soil samples}

Four topsoil-litter mixed samples were collected in the spring of 2017 from the Wolfheze forest in the Netherlands (Additional file 1: Supplementary Table S12). Samples were taken from topsoil $(0$ to $5 \mathrm{~cm})$ adjacent to fallen tree trunks. The collected samples were pooled, sieved (2-mm mesh), and immediately used for SIP incubation with EPS from Granulicella sp. strain WH15 (WH15EPS). The physicochemical properties of the topsoil-litter samples were determined (Eurofins Agro BV, Wageningen, NL) and are presented in Additional file 1: Supplementary Table S13. A workflow diagram of the experiments is depicted in Fig. 7.

\section{SIP metagenome \\ $\left.{ }^{13} \mathrm{C}\right]$-labeled and unlabeled EPS production}

Granulicella sp. strain WH15 was cultivated on PSY5 solid medium [54] containing $3 \%$ (wt/vol) fully ${ }^{13} \mathrm{C}$-labeled glucose as the sole carbon source or unlabeled glucose for unlabeled control EPS production. After 30 days of incubation at $20^{\circ} \mathrm{C}$ polysaccharide portion of EPS was extracted and purified according to Liu et al. [55]. Sixty microliters of $36.5 \%$ formaldehyde was added to each sample and incubated at $4{ }^{\circ} \mathrm{C}$ for $1 \mathrm{~h}$. Next, $4 \mathrm{ml}$ of $1 \mathrm{M}$ $\mathrm{NaOH}$ was added and incubated at $4{ }^{\circ} \mathrm{C}$ for $3 \mathrm{~h}$. After 


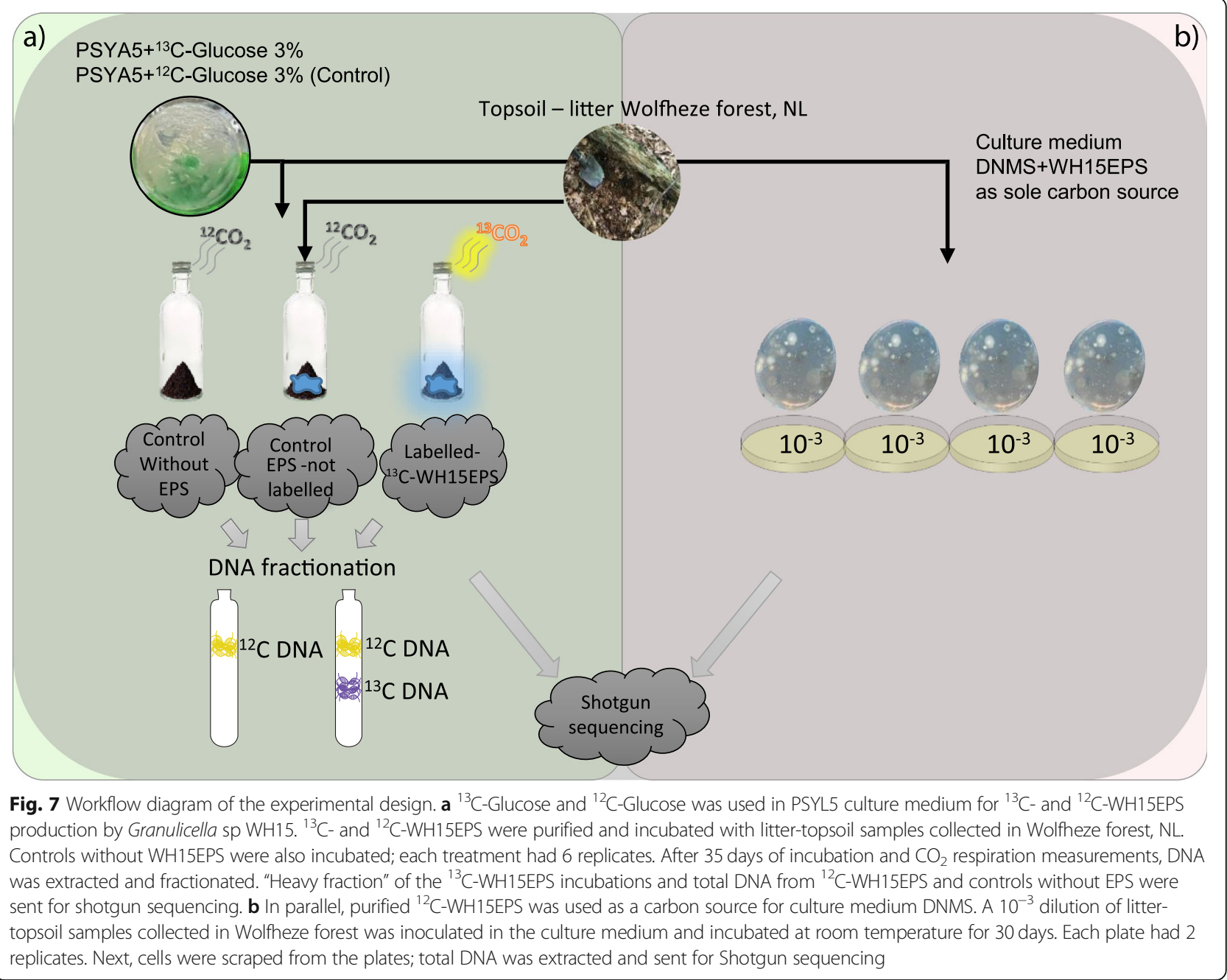

centrifugation at $9000 \times g$ for $40 \mathrm{~min}$, cell debris in the supernatant were eliminated through filtering $(0.2 \mu \mathrm{m}$ membranes, Millipore) at room temperature, and monosaccharides were removed by dialysis in SnakeSkin $^{\text {TM }}$ Dialysis Tubing (3500 Da) (Thermo Fisher Scientific, MA, USA) against demineralized water at $4{ }^{\circ} \mathrm{C}$ for $48 \mathrm{~h}$. DNA concentration in the EPS solution was determined in a Qubit fluorometer using a broadrange Quant-i $\mathrm{T}^{\mathrm{Tm}}$ dsDNA Assay Kit (Invitrogen, Carlsbad, CA, USA). EPS protein concentrations were determined by a Pierce ${ }^{\mathrm{m} x}$ Modified Lowry Protein Assay Kit (Thermo Fisher Scientific, MA, USA). The total carbohydrate content was estimated by the phenolsulfuric acid method [56] modified for 96-well plates [57] with glucose as the standard. The EPS solutions were freeze-dried at $-80{ }^{\circ} \mathrm{C}$ for $72 \mathrm{~h}$ until further processing. The purified EPS contained $\sim 400 \mathrm{mg} / \mathrm{ml}$ carbohydrates, $\sim 1 \%$ protein, and undetectable amounts of DNA.

\section{Stable isotope probing (SIP) incubation}

Freeze-dried EPS was hydrated with $1 \mathrm{ml}$ of Milli-Q sterile water immediately before inoculation in topsoil-litter samples to create a homogeneous distribution. Five grams (wet weight) of topsoil-litter samples with $0.05 \%$ (wt/wt) WH15EPS (labeled and unlabeled controls) or without EPS were added to a $120-\mathrm{ml}$ bottle, which was sealed with a butyl rubber stopper and incubated at room temperature $\left(22{ }^{\circ} \mathrm{C}\right)$ in the dark. Each treatment (labeled EPS, unlabeled EPS, and control without EPS) had six replicates. In order to maintain oxic conditions and prevent ${ }^{13} \mathrm{CO}_{2}$ cross-feeding, all vials were uncapped and aired every 4 days. The use of WH15EPS by the microbial community was monitored as $\mathrm{CO}_{2}$ respiration through gas chromatography (GC) (Trace GC Ultra, Thermo Fisher Scientific, MA, USA), performed daily to monitor the vial headspace $\mathrm{CO}_{2}$. For incubations with $\left[{ }^{13} \mathrm{C}\right]$-labeled EPS, monitoring of the headspace $\mathrm{CO}_{2}$ ${ }^{13} \mathrm{C} /{ }^{12} \mathrm{C}$ ratio was performed via $\mathrm{GC}$ combustion isotope 
ratio mass spectrometry (GC/C/IRMS) (GC IsoLink $\mathrm{II}^{\mathrm{m}}$ IRMS System, Thermo Fisher Scientific, MA, USA). $\mathrm{CO}_{2}$ emissions throughout the experiment are shown in Additional file 1: Supplementary Figure S9. After 35 days of incubation, $0.5 \mathrm{~g}$ of samples were removed from the vials for DNA extraction.

\section{DNA extraction and fractionation}

DNA was extracted from $250 \mathrm{mg}$ of soil with or without ${ }^{13} \mathrm{C}$-labeled/unlabeled substrates with the PowerSoil $^{\circ}$ DNA Isolation Kit (MO BIO Laboratories, Inc) according to the manufacturer's instructions and quantified by a spectrophotometer (NanoDrop ${ }^{\mathrm{Tx}} 2000$, Thermo Fisher Scientific, MA, USA). Gradient fractionation was performed according to Neufeld et al. [58]. Two microgram of DNA were combined with $\mathrm{CsCl}(1.72 \mathrm{~g} / \mathrm{ml})$ and gradient buffer $(100 \mathrm{mM}$ Tris$\mathrm{HCl} \mathrm{pH} \mathrm{8.0,} 100 \mathrm{mM} \mathrm{KCl,} 1 \mathrm{mM}$ EDTA) in an ultracentrifugation tube (PA UltraCrimp $1.8 \mathrm{ml}$, ThermoFisher Scientific, MA, USA) and ultracentrifuged at $125,395 \times g$ (Discovery 120SE ultracentrifuge, ThermoFisher Scientific, Massachusetts, USA) under vacuum at $20{ }^{\circ} \mathrm{C}$ for $65 \mathrm{~h}$. Gradient fractionation resulted in 18 DNA fractions of approximately $100 \mu \mathrm{l}$ each, which density was measured with a refractometer (AR200, Reichert Technologies, New York, USA). DNA was precipitated from the $\mathrm{CsCl}$ with polyethylene glycol solution (30\% PEG6000, $1.6 \mathrm{M} \mathrm{NaCl}$ ) and glycogen $(20 \mu \mathrm{g} / \mu \mathrm{l})$, washed with $70 \%$ ethanol, and eluted in $30 \mu \mathrm{l}$ of $10 \mathrm{mM}$ Tris- $\mathrm{HCl}$ buffer, $\mathrm{pH}$ 8.0. The DNA concentration of each fraction was determined in a Qubit 4 Fluorometer (ThermoFisher Scientific, MA, USA) using a Quant-iT ${ }^{\mathrm{mm}}$ dsDNA HS Assay Kit (Invitrogen, Carlsbad, CA, USA). The unlabeled substrate incubations were used as controls to determine the expected position of labeled soil DNA in the $\mathrm{CsCl}$ density gradients.

Library preparation and high-throughput shotgun sequencing were performed using the "heavy" DNA fractions pooled within each sample replicate as well as the total DNA of both the ${ }^{12} \mathrm{C}$-EPS-amended and unamended controls. Library preparation and Illumina MiSeq PE250 shotgun sequencing were performed at McGill University and Génome Québec Innovation Centre (Montréal, Québec, Canada). The sequences were deposited in the European Nucleotide Archive (ENA; https://www.ebi.ac.uk/ ena) under the accession number PRJEB31257.

\section{Metagenome of cultivated microorganisms in media with WH15EPS as sole carbon source}

For evaluation of the metagenome of microorganisms that were able to grow in culture medium with WH15 EPS as a sole carbon source, $10 \mathrm{~g}$ of fresh topsoil-litter sample were mixed with $100 \mathrm{ml}$ of $100 \mathrm{mM}$ MES buffer
(2-[N-morpholino]ethanesulphonic acid, $1.95 \mathrm{~g} / \mathrm{l}, \mathrm{pH}$ 5.5), agitated for $30 \mathrm{~min}$ at room temperature on a vortex and decanted for $30 \mathrm{~min}$. Dilutions $\left(10^{-3}\right.$ to $\left.10^{-6}\right)$ were prepared in sterile MES buffer, and $200 \mu$ l of the dilutions were plated in quadruplicate. Diluted culture medium DNMS $\left[\mathrm{MgSO}_{4} .7 \mathrm{H}_{2} \mathrm{O} \quad 0.2 \mathrm{~g} / \mathrm{l}, \quad \mathrm{CaCl}_{2} .2 \mathrm{H}_{2} \mathrm{O}\right.$ $0.053 \mathrm{~g} / \mathrm{l}$, chelated iron solution $0.2 \mathrm{ml} / \mathrm{l}$ (ferric III ammonium citrate $0.1 \mathrm{~g} / 100 \mathrm{ml}$, EDTA $0.2 \mathrm{~g} / 100 \mathrm{ml}, \mathrm{HCl}$ $0.3 \mathrm{ml} / 100 \mathrm{ml}$ ) trace element solution SL10 $1 \mathrm{ml} / \mathrm{L}$ [59], $\mathrm{NH}_{4} \mathrm{Cl} 0.1 \mathrm{~g} / \mathrm{l}$, agar $20 \mathrm{~g} / \mathrm{l}$ ] with added WH15EPS [23] $(0.05 \%) \mathrm{pH} 5.5$ and $40 \mathrm{ng} / \mu \mathrm{l}(40 \mathrm{mg} / \mathrm{l})$ cicloheximide to prevent growth of fungi was used for plating. To prevent caramelization, the freeze-dried purified WH15EPS was hydrated with Milli-Q water, sterilized by filtration through a $0.2 \mu \mathrm{m}$ membrane (Millipore), and added to the culture medium after autoclaving. Chelated iron solution and trace element solution SL10 were added after autoclaving and cooling of the culture medium. The plates inoculated with the soil suspension were incubated at room temperature for 1 month. The dilution $10^{-3}$ was chosen for sequencing. After incubation, colonies were scraped and used for total DNA extraction with PowerSoil ${ }^{\circ}$ DNA Isolation Kit (MO BIO Laboratories, Inc). Following the first DNA extraction, a second round of DNA extraction was performed for each sample, according to Dimitrov et al. [60]. The total DNA extracted from the plates was used for metagenome shotgun sequencing. Library preparation and Illumina HiSeq XTen sequencing were performed at Genewiz (Suzhou, China). The sequences were deposited in the European Nucleotide Archive (ENA; https://www.ebi.ac. uk/ena) under the accession number PRJEB24069.

\section{Bioinformatics and statistical analyses of metagenome data SIP metagenome}

SIP metagenome sequences were processed using EBI MGnify [61] pipeline and SqueezeMeta [62] pipeline in sequential mode. Briefly, in the SqueezeMeta pipeline, trimming and quality filtering were performed using Trimmomatic [63]; assembly for each sample separately was done using Megahit [64]; Prodigal [65] was used for ORF prediction, and barrnap [66] was employed for rRNA gene sequence retrieval, which were classified using RDP classifier [67]. Diamond [68] software was used for taxonomic classification of the ORFs against Genbank nr database and functional annotation with eggNOG database, for KO and COG numbers [69]. eggNOG-mapper [70] was employed for carbohydrateactive enzymes annotation with against dbCAN [71]. SqueezeMeta script SQM2tables.py was used to compute the average coverage and normalized TPM (transcripts per million) values for information on gene and function abundances. Normalized TPM SqueezeMeta ORF 
dataset and 16S rRNA gene data recovered from MGNify analysis were used for statistical analyses, performed in RStudio version 1.1.423 running $\mathrm{R}$ version 3.5.1 [72]. For the 16S-based analysis, OTUs with less than 1 count across all the samples, chloroplast and mitochondrial sequences were discarded; prior to alpha diversity analyses, the data were rarefied to the size of the smallest sample (175 reads). For both ORF-based and $16 \mathrm{~S}$ gene-based taxonomy datasets, "Phyloseq" package [73] was used to calculate the number of observed OTUs, Shannon and Inverse Simpson diversity indices, and Chao1 and ACE diversity estimators. Significant differences in the estimators between treatments were evaluated through parametric and non-parametric tests, including ANOVA, Kruskal-Wallis, and Tukey's HSD tests (package "agricolae") [74]. Bray-Curtis distance matrices constructed using the Hellinger transformed [75] datasets were used for principal coordinate analysis (PCoA) using the capscale function from the "vegan" package v. 2.4.6 [76]. Group dissimilarities were tested by permutational multivariate analysis of variance (PERMANOVA) using the function Adonis from the "vegan" package. CANOCO (version5) [77] was employed to explore the relationship between sample treatments and taxa abundance through redundancy analysis (RDA) in the Hellinger transformed datasets. The statistical significance $(p$ value $<0.05)$ of eigenvalues and treatmenttaxa abundance correlations was tested using Monte Carlo permutation test at 499 permutations, and the top 20 taxa associated with the dispersion of the treatments were displayed in RDA graphs.

In order to identify predicted functions (COG, KEGG, and CAZYmes) responsible for the observed clustering patterns, we performed a feature selection using a "random forest" algorithm using the $\mathrm{R}$ package Boruta [78] (1,000 trees, $p$ value $<0.05)$. Boruta tests if the importance of each individual variable is significantly higher that the importance of a random variable by fitting random forest models iteratively until all predictor variables are classified as "confirmed" or "rejected" at the 0.05 alpha level [79]. The heatmaps for relevant features for each function were constructed with pheamap [80] $\mathrm{R}$ package, based on $z$-score transformed TPM (transcripts per million) abundances to improve normality and homogeneity of the variances. Sequences were submitted to the European Nucleotide Archive (ENA) and are available under the accession number PRJEB31257.

\section{Metagenome analysis for cultivated microorganisms}

The DNA of the cultivated microorganisms were shotgun metagenome sequenced, and the sequences were processed using EBI MGnify [61] pipeline and ATLAS (Automatic Tool for Local Assembly Structures) [81] pipeline. For ATLAS, quality filtering was performed using BBDuk2, and cross-assembly was done with Megahit [64]; functional and taxonomic analysis were performed at ORF level for the assembled contigs. Prodigal [65] was used for ORF prediction, and eggNOG database [69] was used for functional annotation (COG and KO numbers) using the DIAMOND software [68]. eggNOGmapper [70] was used for functional annotation of CAZymes with dbCAN [71]. The Kaiju software [82] was used for ORF taxonomy assignment against NCBI RefSeq database. Custom scripts were used to generate tables containing information of taxonomy and function abundance of the ORFs in all samples. Quality controlled contigs $>1000 \mathrm{~kb}$ were used for binning using Concoct [83], Maxbin [84], and Metabat [85]; resulting bins were refined using DAS tool [86], and genome dereplication was performed with dRep [87]. Completeness and contamination of the assembled genomes were checked using CheckM [88], as well as taxonomy assignment. The ORFs of the genomes were predicted using Prodigal [65], and DIAMOND software [68] was used for functional annotation with eggNOG (COG and KO numbers) [69]. The annotation of CAZYmes was performed with eggNOG-mapper [70] against dbCAN [71]. Sequences were submitted to the European Nucleotide Archive (ENA) and are available under the accession number PRJEB24069.

\section{Supplementary information}

Supplementary information accompanies this paper at https://doi.org/10. 1186/s40168-020-00836-7.

\footnotetext{
Additional file 1 Supplementary Figure S1: Taxonomic composition and relative abundance of microbial groups at phylum level in SIP metagenome treatments based on a) SSU rRNA gene sequence classification ( $>2.2 \%$ abundance) b) ORF taxonomic classification $(>0.1 \%$ abundance). Average abundances of 4 replicates. Unc: unclassified. No EPS - incubation without WH15EPS. Unlab EPS-incubation containing ${ }^{12} \mathrm{C}-$ WH15EPS. Heavy - 'heavy fraction' of incubations containing ${ }^{13} \mathrm{C}$ WH15EPS; Supplementary Figure S2: Biplot of the Redundancy analysis (RDA) based on normalized and Hellinger-transformed abundances of a) SSU rRNA gene taxonomy classification and b) ORF taxonomic classification. Only the best 20 fitting groups are displayed. Unc: unclassified. No EPS - incubation without WH15EPS. Unlab EPS-incubation containing ${ }^{12} \mathrm{C}-$ WH15EPS. Heavy - 'heavy fraction' of incubations containing ${ }^{13} \mathrm{C}$ WH15EPS; Supplementary Figure S3: Box-plot comparisons of alphadiversity assessment by richness estimators (number of observed OTUs, Chao1, ACE) and diversity indices (Shannon, Inverse Simpson) for SIP 165 rRNA gene samples. 'Heavy fraction' values are significantly lower in comparison with both controls for all comparisons ( $p$-value $<0.05$ ). Comparisons performed across treatments using ANOVA test and Tukey's HSD post-hoc test. Data rarefied to the minimum sampling depth. Unlab. EPSincubation containing ${ }^{12} \mathrm{C}-\mathrm{WH} 15 \mathrm{EPS}$. Heavy - 'heavy fraction' of incubations containing ${ }^{13} \mathrm{C}-\mathrm{WH} 15 \mathrm{EPS}$; Supplementary Figure S4: Relative abundance distribution of the most abundant functional categories in TPM-normalized metagenome sequencing data from the SIP metagenome. a) COG annotation (all categories); b) KEGG annotation (above $0.1 \%$ abundance); c) dbCAN annotation (above 1\% abundance). E-Amino acid transport and metabolism; G- Carbohydrate transport and metabolism; $\mathrm{H}$ Coenzyme transport and metabolism; C-Energy production and conversion; I-Lipid transport and metabolism; F-Nucleotide transport and
} 
metabolism; Q- Secondary metabolites; D-Cell acycle; N-Cell motility; MCell wall/membrane/envelope biogenesis; V-Defence mechanisms; PInorganic ion transport and metabolism; U-Intracellular trafficking; O-Post translational modification; T-Signal transduction mechanisms; LReplication, recombination and repair; K-Transcription; J-Translation; SFunction unknown; R-General function and prediction; X-Mobilome.; Supplementary Figure S5: Principal Coordinate Analysis (PCOA) clustering of normalized and Hellinger-transformed SIP metagenome sequencing data based on Bray-Curtis distances of a) COG annotation, b) KEGG annotation and c) dbCAN annotation. No EPS - incubation without WH15EPS. Unlabeled EPS-incubation containing ${ }^{12} \mathrm{C}-$ WH15EPS. Heavy - 'heavy fraction' of incubations containing ${ }^{13} \mathrm{C}-\mathrm{WH} 15 \mathrm{EPS}$; Supplementary Figure S6: Taxonomic composition and relative abundance of microbial groups at a) kingdom and b) phylum level in samples from the metagenome shotgun of cultivated microrganims based SSU rRNA gene taxonomic classification. Average from 2 replicates per plate of culture medium.; Supplementary Figure S7: Venn diagram depicting the number of common and unique glycoside hydrolase (GH) families observed in SIP metagenome and metagenome of cultivate microorganisms ' datasets; Supplementary Figure S8: Distribution of the 20 most abundant CAZyme families in a) SIP metagenome samples (relative abundance, average of 4 replicates); b) metagenome of cultivated microorganisms (relative abundance, average of 2 replicates); c) Metagenome-Assembled Genomes (MAGs) (number of genes), and most abundant glycosyl hydrolases (GH) in d) SIP metagenome samples (relative abundance, average of 4 replicates), e) metagenome of cultivated microorganisms (relative abundance, average of 2 replicates) and f) Metagenome-Assembled Genomes (MAGs) (number of genes); Supplementary Figure S9: $\mathrm{CO}_{2}$ emission. $\mathrm{CO}_{2}$ production during total incubation period. Control: control without EPS; EPS: control containing ${ }^{12} \mathrm{C}$-EPS; Labeled: incubation with ${ }^{13} \mathrm{C}$-EPS; Labeled $\mathrm{CO}_{2}$ percentage: ${ }^{13} \mathrm{CO}_{2}$ emitted during ${ }^{13} \mathrm{C}$-EPS sample incubation; water: days when samples were hydrated; air: days when samples were aired. Supplementary Table S1: COG functions that significantly segregated across treatments selected by Boruta random forests algorithm based on 1000 permutations in the SIP metagenome treatment comparisons; Supplementary Table S2: KEGG orthologs that significantly segregated across treatments selected by Boruta random forests algorithm based on 1000 permutations in the SIP metagenome treatment comparisons; Supplementary Table S3:CAZyme families that significantly segregated across treatments selected by Boruta random forests algorithm based on 1000 permutations in the SIP metagenome treatment comparisons; Supplementary Table S4: Most abundant CAZyme families (above 1\% abundance) and most abundant KEGG orthologs (above 0.2\% abundance) in the shotgun metagenome of cultivated microorganisms; Supplementary Table S5: MAGs coverage in all samples; Supplementary Table S6: Most abundant KEGG orthologs in MAGs and their associated functions. A selection of the top 10 most abundant KEGG orthologs in each genome is displayed. Annotation performed using eggNOG database.; Supplementary Table S7: Sugar transporters in MAG1 annotated with eggNOG database; Supplementary Table S8: Sugar transporters in MAG2 annotated with eggNOG database; Supplementary Table S9: General type transporters in MAG3 annotated with eggNOG database; Supplementary Table S10: Sugar transporters in MAG4 annotated with eggNOG database; Supplementary Table S11: Families of CAZymes observed in the MAGs, number of ORFs and associated functions; Supplementary Table S12: Coordinates of the sampling sites; Supplementary Table S13: Physicochemical properties of topsoil-litter samples.

Acknowledgements

We would like to thank Wietse de Boer for helping with the sampling local.

\section{Authors' contributions}

O.Y.A.C. and E.E.K designed research; O.Y.A.C and A.P. conducted the experiment; O.Y.A.C and A.P. conducted the DNA extraction; B.L. prepared the metagenome libraries and sequenced the samples. O.Y.A.C. and M.H. performed the bioinformatics and statistical analyses; O.Y.A.C., B.L., and E.E.K wrote the paper. All authors read and approved the final manuscript.

\section{Funding}

This research was supported by The Netherlands Organization for Scientific Research (NWO) 729.004.003. Ohana Y.A. Costa was supported by an SWB grant from CNPq [202496/2015-5] (Conselho Nacional de Desenvolvimento Científico e Tecnológico). B.L. was supported by the Hundred Talents Program of The Chinese Academy of Sciences. Publication number 6945 of the Netherlands Institute of Ecology (NIOO-KNAW).

\section{Availability of data and materials}

The sequences were deposited in the European Nucleotide Archive (ENA; https://www.ebi.ac.uk/ena) under the accession numbers PRJEB24069 and PRJEB31257.

Ethics approval and consent to participate

Not applicable.

\section{Competing interests}

The authors declare that they have no competing interests.

\section{Author details}

${ }^{1}$ Netherlands Institute of Ecology (NIOO-KNAW), Department of Microbial Ecology, Droevendaalsesteeg 10, 6708 PB Wageningen, Netherlands. ${ }^{2}$ Institute of Biology (IBL), Leiden University, Leiden, The Netherlands. ${ }^{3}$ Center for Agricultural Resources Research, Institute of Genetics and Developmental Biology, Chinese Academy of Sciences, Shijiazhuang 050021, Hebei, China.

Received: 7 February 2020 Accepted: 31 March 2020

Published online: 01 June 2020

\section{References}

1. Verastegui Y, Cheng J, Engel K, Kolczynski D, Mortimer S, Lavigne J, Montalibet J, Romantsov T, Hall M, McConkey BJ et al: Multisubstrate isotope labeling and metagenomic analysis of active soil bacterial communities. mBio. 2014;5(4):e01157-e01114. https://doi.org/10.1128/mBio. 01157-14.

2. Nahar S, Mizan MFR, Ha AJ-W, Ha S-D: Advances and future prospects of enzyme-based biofilm prevention approaches in the food industry. Compr Rev Food Sci Food Saf 2018;17(6):1484-1502. https://doi.org/10.1111/15414337.12382.

3. Madhavan A, Sindhu R, Parameswaran B, Sukumaran RK, Pandey A: Metagenome analysis: a powerful tool for enzyme bioprospecting. Appl Biochem Biotech 2017;183(2):636-651. https://doi.org/10.1007/s12010-0172568-3.

4. Berini F, Casciello C, Marcone GL, Marinelli F: Metagenomics: novel enzymes from non-culturable microbes. FEMS Microbiol Lett. 2017;364(21). https:// doi.org/10.1093/femsle/fnx211.

5. Ferrer M, Martínez-Martínez M, Bargiela R, Streit WR, Golyshina OV, Golyshin PN: Estimating the success of enzyme bioprospecting through metagenomics: current status and future trends. Microb Biotechnol. 2016; 9(1):22-34. https://doi.org/10.1111/1751-7915.12309.

6. Zhao C, Chu Y, Li Y, Yang C, Chen Y, Wang X, Liu B: High-throughput pyrosequencing used for the discovery of a novel cellulase from a thermophilic cellulose-degrading microbial consortium. Biotechnol Lett 2016;39(1):123-131. https://doi.org/10.1007/s10529-016-2224-y.

7. Bergmann JC, Costa OYA, Gladden JM, Singer S, Heins R, D'Haeseleer P, Simmons BA, Quirino BF: Discovery of two novel $\beta$-glucosidases from an Amazon soil metagenomic library. FEMS Microbiol Lett. 2014;351(2):147-155. https://doi.org/10.1111/1574-6968.12332.

8. Coughlan LM, Cotter PD, Hill C, Alvarez-Ordóñez A: Biotechnological applications of functional metagenomics in the food and pharmaceutical industries. Front Microbiol 2015;6. https://doi.org/10.3389/fmicb.2015.00672.

9. Ezeilo UR, Zakaria II, Huyop F, Wahab RA: Enzymatic breakdown of lignocellulosic biomass: the role of glycosyl hydrolases and lytic polysaccharide monooxygenases. Biotechnol Biotechnol Equip 2017:1-16. https://doi.org/10.1080/13102818.2017.1330124.

10. Costa OYA, Raaijmakers JM, Kuramae EE: Microbial extracellular polymeric substances: ecological function and impact on soil aggregation. Front Microbiol. 2018;9:1636. https://doi.org/10.3389/fmicb.2018.01636.

11. Flemming $\mathrm{H}-\mathrm{C}$, Wingender J: The biofilm matrix. Nat Rev Microbiol. 2010: 623-633. https://doi.org/10.1038/nrmicro2415. 
12. Liu X, Kokare C. Microbial enzymes of use in industry. In: Biotechnology of microbial enzymes; 2017. p. 267-98.

13. Legin E, Ladrat C, Godfroy A, Barbier G, Duchiron F: Thermostable amylolytic enzymes of thermophilic microorganisms from deep-sea hydrothermal vents. Comptes Rendus Acad Sci 1997;320(11):893-898. https://doi.org/10. 1016/s0764-4469(97)80874-8.

14. Oh HN, Park D, Seong HJ, Kim D, Sul WJ: Antarctic tundra soil metagenome as useful natural resources of cold-active lignocelluolytic enzymes. J Microbiol 2019;57(10):865-873. https://doi.org/10.1007/s12275019-9217-1.

15. Hess M, Sczyrba A, Egan R, Kim TW, Chokhawala H, Schroth G, Luo S, Clark DS, Chen F, Zhang T et al: Metagenomic discovery of biomass-degrading genes and genomes from cow rumen. Science. 2011;331(6016):463-467. https://doi.org/10.1126/science.1200387.

16. Warnecke F, Luginbühl $P$, Ivanova N, Ghassemian M, Richardson TH, Stege JT, Cayouette M, McHardy AC, Djordjevic G, Aboushadi N et al: Metagenomic and functional analysis of hindgut microbiota of a woodfeeding higher termite. Nature. 2007;450(7169):560-565. https://doi.org/10. 1038/nature06269.

17. Vivanco L, Rascovan N, Austin AT: Plant, fungal, bacterial, and nitrogen interactions in the litter layer of a native Patagonian forest. PeerJ 2018;6. https://doi.org/10.7717/peerj.4754.

18. Sista Kameshwar AK, Qin W: Comparative study of genome-wide plant biomass-degrading CAZymes in white rot, brown rot and soft rot fungi. Mycology 2017;9(2):93-105. https://doi.org/10.1080/21501203.2017.1419296.

19. Chen Y, Pu G, Lian B, Pei X, Huang G, Wang Q, Lv Y: Interactions between two fungi strains during litter decomposition through a microcosm experiment: different degradative enzyme activities. Adv Enzyme Res 2018; 06(01):1-9. https://doi.org/10.4236/aer.2018.61001.

20. Baldrian $P$, Kolařík M, Štursová M, Kopecký J, Valášková V, Větrovský T, Žifčáková L, Šnajdr J, Rídl J, Vlček Č et al: Active and total microbial communities in forest soil are largely different and highly stratified during decomposition. ISME J. 2011;6(2):248-258. https://doi.org/10.1038/ismej.2011. 95.

21. Kielak AM, Barreto CC, Kowalchuk GA, van Veen JA, Kuramae EE: The ecology of acidobacteria: moving beyond genes and genomes. Front Microbiol 2016;7: 16. https://doi.org/10.3389/fmicb.2016.00744.

22. Rawat SR, Männistö MK, Starovoytov V, Goodwin L, Nolan M, Hauser L, Land M, Davenport KW, Woyke T, Häggblom MM: Complete genome sequence of Granulicella mallensis type strain MP5ACTX8T, an acidobacterium from tundra soil. Stand Genomic Sci 2013;9(1):71-82. https:// doi.org/10.4056/sigs.4328031.

23. Kielak AM, Castellane TCL, Campanharo JC, Colnago LA, Costa OYA, Corradi da Silva ML, van Veen JA, Lemos EGM, Kuramae EE: Characterization of novel Acidobacteria exopolysaccharides with potential industrial and ecological applications. Sci Rep. 2017;7:41193. https://doi.org/10.1038/ srep41193.

24. Rehm BHA: Bacterial polymers: biosynthesis, modifications and applications. Nat Rev Microbiol. 2010;8(8):578-592. https://doi.org/10.1038/nrmicro2354.

25. Neufeld JD, Wagner M, Murrell JC: Who eats what, where and when? Isotope-labelling experiments are coming of age. ISME J 2007;1 (2):103-110. https://doi.org/10.1038/ismej.2007.30.

26. Ginige MP, Hugenholtz P, Daims H, Wagner M, Keller J, Blackall LL: Use of stable-isotope probing, full-cycle rRNA analysis, and fluorescence in situ hybridization-microautoradiography to study a methanol-fed denitrifying microbial community. Appl Environ Microbiol 2004;70(1):588-596. https:// doi.org/10.1128/aem.70.1.588-596.2004.

27. Padmanabhan P, Padmanabhan S, DeRito C, Gray A, Gannon D, Snape JR, Tsai CS, Park W, Jeon C, Madsen EL: Respiration of 13C-labeled substrates added to soil in the field and subsequent 16S rRNA gene analysis of 13clabeled soil DNA. Appl Environ Microbiol 2003;69(3):1614-1622. https://doi. org/10.1128/aem.69.3.1614-1622.2003.

28. Li J, Zhang D, Song M, Jiang L, Wang Y, Luo C, Zhang G: Novel bacteria capable of degrading phenanthrene in activated sludge revealed by stableisotope probing coupled with high-throughput sequencing. Biodegradation. 2017;28(5-6):423-436. https://doi.org/10.1007/s10532-017-9806-9.

29. Pinnell LJ, Dunford E, Ronan P, Hausner M, Neufeld JD: Recovering glycoside hydrolase genes from active tundra cellulolytic bacteria. Can J Microbiol. 2014;60(7):469-476. https://doi.org/10.1139/cjm-2014-0193.

30. de Castro VHL, Schroeder LF, Quirino BF, Kruger RH, Barreto CC: Acidobacteria from oligotrophic soil from the Cerrado can grow in a wide range of carbon source concentrations. Can J Microbiol 2013;59(11):746-753. https://doi.org/10.1139/cjm-2013-0331.

31. Haichar FeZ, Achouak W, Christen R, Heulin T, Marol C, Marais M-F, Mougel C, Ranjard L, Balesdent J, Berge O: Identification of cellulolytic bacteria in soil by stable isotope probing. Environ Microbiol 2007;9(3):625-634. https:// doi.org/10.1111/j.1462-2920.2006.01182.x.

32. Ivanova AA, Wegner C-E, Kim Y, Liesack W, Dedysh SN: Metatranscriptomics reveals the hydrolytic potential of peat-inhabiting Planctomycetes. Antonie Van Leeuwenhoek. 2017;111(6):801-809. https://doi.org/10.1007/s10482-0170973-9.

33. Staley JT, Konopka A: Measurement of in situ activities of nonphotosynthetic microorganisms in aquatic and terrestrial habitats. Annu Rev Microbiol. 1985;39(1):321-346. https://doi.org/10.1146/annurev.mi.39. 100185.001541 .

34. Vester JK, Glaring MA, Stougaard P: Improved cultivation and metagenomics as new tools for bioprospecting in cold environments. Extremophiles. 2015; 19(1):17-29. https://doi.org/10.1007/s00792-014-0704-3.

35. Schmid J, Heider D, Wendel NJ, Sperl N, Sieber V: Bacterial glycosyltransferases: challenges and opportunities of a highly diverse enzyme class toward tailoring natural products. Front Microbiol. 2016;7. https://doi.org/10.3389/fmicb.2016.00182.

36. Lairson LL, Henrissat B, Davies GJ, Withers SG: Glycosyltransferases: structures, functions, and mechanisms. Annu Rev Biochem. 2008;77(1):521555. https://doi.org/10.1146/annurev.biochem.76.061005.092322.

37. Liang D-M, Liu J-H, Wu H, Wang B-B, Zhu H-J, Qiao J-J: Glycosyltransferases: mechanisms and applications in natural product development. Chem Soc Rev 2015;44(22):8350-8374. https://doi.org/10.1039/c5cs00600g.

38. Mulichak AM, Losey HC, Lu W, Wawrzak Z, Walsh CT, Garavito RM: Structure of the TDP-epi-vancosaminyltransferase GtfA from the chloroeremomycin biosynthetic pathway. Proc Natl Acad Sci U S A. 2003;100(16):9238-9243. https://doi.org/10.1073/pnas.1233577100.

39. Mulichak AM, Lu W, Losey HC, Walsh CT, Garavito RM: Crystal structure of vancosaminyltransferase GtfD from the vancomycin biosynthetic pathway: interactions with acceptor and nucleotide ligands. Biochemistry. 2004;43(18) 5170-5180. https://doi.org/10.1021/bi036130c.

40. Moncrieffe MC, Fernandez M-J, Spiteller D, Matsumura H, Gay NJ, Luisi BF, Leadlay PF: Structure of the glycosyltransferase EryCIII in complex with its activating P450 homologue EryCll. J Mol Biol. 2012;415(1):92-101. https://doi. org/10.1016/j.jmb.2011.10.036.

41. Labes A, Karlsson EN, Fridjonsson OH, Turner P, Hreggvidson GO, Kristjansson JK, Holst O, Schonheit P: Novel members of glycoside hydrolase family 13 derived from environmental DNA. Appl Environ Microbiol 2008; 74(6):1914-1921. https://doi.org/10.1128/aem.02102-07.

42. Fleming D, Chahin L, Rumbaugh K: Glycoside hydrolases degrade polymicrobial bacterial biofilms in wounds. Antimicrob Agents Chemother 2016. https://doi.org/10.1128/aac.01998-16.

43. Craigen B: The use of commercially available alpha-amylase compounds to inhibit and remove Staphylococcus aureus biofilms. Open Microbiol J. 2011; 5(1):21-31. https://doi.org/10.2174/1874285801105010021.

44. Liu QP, Sulzenbacher G, Yuan H, Bennett EP, Pietz G, Saunders K, Spence J, Nudelman E, Levery SB, White T et al: Bacterial glycosidases for the production of universal red blood cells. Nature Biotechnol. 2007;25(4):454464. https://doi.org/10.1038/nbt1298.

45. Fu XT, Kim SM: Agarase: review of major sources, categories, purification method, enzyme characteristics and applications. Mar Drugs. 2010;8(1):200218. https://doi.org/10.3390/md8010200.

46. Khan RH, Du L, Pang H, Wang Z, Lu J, Wei Y, Huang R: Characterization of an invertase with $\mathrm{pH}$ tolerance and truncation of its $\mathrm{N}$-terminal to shift optimum activity toward neutral pH. PLoS One. 2013;8(4). https://doi.org/10. 1371/journal.pone.0062306.

47. Mohan A, Flora B, Girdhar M. Inulinase: an important microbial enzyme in food industry. In: Microbial bioprospecting for sustainable development; 2018. p. 237-48.

48. Wiater A, Szczodrak J, Pleszczynska M, Próchniak K: Production and use of mutanase from Trichoderma harzianum for effective degradation of streptococcal mutans. Braz J Microbiol. 2005;36(2). https://doi.org/10.1590/ s1517-83822005000200008

49. Konan HK, Yapi D, Bi CYY, Koné TFM, Kouadio PEJN, Patrice K. Biochemical characterization of two acid phosphatases purified from breadfruit (Artocarpus communis) seeds. J Adv Biol Biotechnol. 2016:43: 1102-13. 
50. Hukić M, Seljmo D, Ramovic A, Ibrišimović MA, Dogan S, Hukic J, Bojic EF: The effect of lysozyme on reducing biofilms by Staphylococcus aureus, Pseudomonas aeruginosa, and Gardnerella vaginalis: an in vitro examination. Microb Drug Resist 2018;24(4):353-358. https://doi.org/10.1089/ mdr.2016.0303.

51. Mallela K, Talens-Perales D, Górska A, Huson DH, Polaina J, Marín-Navarro J: Analysis of domain architecture and phylogenetics of family 2 glycoside hydrolases (GH2). PLoS One. 2016;11(12). https://doi.org/10.1371/journal. pone.0168035.

52. Dahiya N, Tewari R, Tiwari RP, Hoondal GS: Chitinase production in solidstate fermentation by Enterobacter sp. NRG4 using statistical experimental design. Curr Microbiol 2005;51(4):222-228. https://doi.org/10.1007/s00284005-4520-y.

53. Helbert W, Poulet L, Drouillard S, Mathieu S, Loiodice M, Couturier M, Lombard V, Terrapon N, Turchetto J, Vincentelli R et al: Discovery of novel carbohydrate-active enzymes through the rational exploration of the protein sequences space. Proc Natl Acad Sci U S A 2019;116(13):6063-6068. https://doi.org/10.1073/pnas.1815791116.

54. Campanharo JC, Kielak AM, Castellane TCL, Kuramae EE, Lemos EGdM: Optimized medium culture for Acidobacteria subdivision 1 strains. FEMS Microbiol Lett. 2016; 363(21):fnw245. https://doi.org/10.1093/femsle/fnw245.

55. Liu H, Fang HH: Extraction of extracellular polymeric substances (EPS) of sludges. J Biotechnol. 2002;95(3):249-256. https://doi.org/10.1016/S01681656(02)00025-1.

56. DuBois M, Gilles KA, Hamilton JK, Rebers PA, Smith F: Colorimetric method for determination of sugars and related substances. Anal Chem. 1956;28(3):350-356. https://doi.org/10.1021/ac60111a017.

57. Masuko T, Minami A, Iwasaki N, Majima T, Nishimura S-I, Lee YC: Carbohydrate analysis by a phenol-sulfuric acid method in microplate format. Anal Biochem. 2005;339(1):69-72. https://doi.org/10.1016/j.ab.2004.12. 001.

58. Neufeld JD, Vohra J, Dumont MG, Lueders T, Manefield M, Friedrich MW Murrell JC: DNA stable-isotope probing. Nat Protoc. 2007;2(4):860-866. https://doi.org/10.1038/nprot.2007.109.

59. Atlas RM: Handbook of microbiological media, 4rd edn. Boca Raton, Florida: CRC Press c 2004.;2010.

60. Dimitrov MR, Veraart AJ, de Hollander M, Smidt H, van Veen JA, Kuramae EE: Successive DNA extractions improve characterization of soil microbial communities. PeerJ 2017;5:e2915. https://doi.org/10.7717/peerj.2915.

61. Mitchell AL, Scheremetjew M, Denise H, Potter S, Tarkowska A, Qureshi M, Salazar GA, Pesseat S, Boland MA, Hunter Fiona M I et al: EBI metagenomics in 2017: enriching the analysis of microbial communities, from sequence reads to assemblies. Nucleic Acids Res 2017;46(D1):D726-D735. https://doi. org/10.1093/nar/gkx967.

62. Tamames J, Puente-Sánchez F: SqueezeMeta, a highly portable, fully automatic metagenomic analysis pipeline. Front Microbiol. 2019;9. https:// doi.org/10.3389/fmicb.2018.03349.

63. Bolger AM, Lohse M, Usadel B: Trimmomatic: a flexible trimmer for Illumina sequence data. Bioinformatics 2014;30(15):2114-2120. https://doi.org/10. 1093/bioinformatics/btu170.

64. Li D, Liu C-M, Luo R, Sadakane K, Lam T-W: MEGAHIT: an ultra-fast singlenode solution for large and complex metagenomics assembly via succinct de Bruijn graph. Bioinformatics. 2015;31(10):1674-1676. https://doi.org/10. 1093/bioinformatics/btv033.

65. Hyatt D, Chen G-L, LoCascio PF, Land ML, Larimer FW, Hauser LJ: Prodigal: prokaryotic gene recognition and translation initiation site identification. BMC Bioinformatics. 2010;11(1). https://doi.org/10.1186/1471-2105-11-119.

66. Seemann T: Basic rapid ribosomal RNA predictor 0.9. 2018. https://github. com/tseemann/barrnap.

67. Wang Q, Garrity GM, Tiedje JM, Cole JR: Naive Bayesian classifier for rapid assignment of rRNA sequences into the new bacterial taxonomy. App Environ Microbiol. 2007;73(16):5261-5267. https://doi.org/10.1128/aem. 00062-07.

68. Buchfink B, Xie C, Huson DH: Fast and sensitive protein alignment using DIAMOND. Nat Methods. 2014;12(1):59-60. https://doi.org/10.1038/nmeth. 3176.

69. Huerta-Cepas J, Szklarczyk D, Forslund K, Cook H, Heller D, Walter MC, Rattei T, Mende DR, Sunagawa S, Kuhn M et al: eggNOG 4.5: a hierarchical orthology framework with improved functional annotations for eukaryotic, prokaryotic and viral sequences. Nucleic Acids Res. 2016; 44(D1):D286-D293. https://doi.org/10.1093/nar/gkv1248.
70. Huerta-Cepas J, Forslund K, Coelho LP, Szklarczyk D, Jensen LJ, von Mering C, Bork P: Fast genome-wide functional annotation through orthology assignment by eggNOG-mapper. Mol Biol Evol. 2017;34(8):2115-2122. https://doi.org/10.1093/molbev/msx148.

71. Yin Y, Mao X, Yang J, Chen X, Mao F, Xu Y: dbCAN: a web resource for automated carbohydrate-active enzyme annotation. Nucleic Acids Res. 2012; 40(W1):W445-W451. https://doi.org/10.1093/nar/gks479.

72. Core R. Team: R: a Language and environment for statistical computing. Vienna: R Foundation for Statistical Computing;2015.

73. McMurdie PJ, Holmes S: phyloseq: an r package for reproducible interactive analysis and graphics of microbiome census data. PLoS One. 2013;8(4). https://doi.org/10.1371/journal.pone.0061217.

74. Mendiburu Fd: Statistical procedures for agricultural research. 2017. https:// CRAN.R-project.org/package=agricolae.

75. Legendre P, Gallagher ED: Ecologically meaningful transformations for ordination of species data. Oecol 2001;129(2):271-280. https://doi.org/10. 1007/s004420100716.

76. Oksanen J, Blanchet FG, Friendly M, Kindt R, Legendre P, McGlinn D, Minchin PR, O'Hara RB, Simpson GL, Solymos P et al: vegan: community ecology package. R package version 2.4-6. 2018. https://CRAN.R-project.org/ package= vegan .

77. Braak CJF, Smilauer P. Canoco reference manual and user's guide: software for ordination, version 5.0. Microcomputer Power: Ithaca USA;2012.

78. Kursa MB, Rudnicki WR: Feature selection with the Boruta package. Stat Softw. 2010;36(11). https://doi.org/10.18637/jss.v036.i11.

79. Leutner BF, Reineking B, Müller J, Bachmann M, Beierkuhnlein C, Dech S, Wegmann M: Modelling forest a-diversity and floristic composition — on the added value of LiDAR plus hyperspectral remote sensing. Remote Sens 2012; 4(9):2818-2845. https://doi.org/10.3390/rs4092818.

80. Kolde R: pheatmap: pretty heatmaps. R package version 1.0.12. 2019. https:// CRAN.R-project.org/package=pheatmap

81. White III RA, Brown J, Colby S, Overall CC, Lee J-Y, Zucker J, Glaesemann KR, Jansson C, Jansson JK: ATLAS (Automatic Tool for Local Assembly Structures) - a comprehensive infrastructure for assembly, annotation, and genomic binning of metagenomic and metatranscriptomic data. PeerJ. 2017;1(e2843). https://doi.org/10.7287/peerj.preprints.2843v1.

82. Menzel $\mathrm{P}, \mathrm{Ng} \mathrm{KL}$, Krogh A: Fast and sensitive taxonomic classification for metagenomics with Kaiju. Nat Commun. 2016;7. https://doi.org/10.1038/ ncomms 11257.

83. Alneberg J, Bjarnason BS, de Bruijn I, Schirmer M, Quick J, ljaz UZ, Lahti L, Loman NJ, Andersson AF, Quince C: Binning metagenomic contigs by coverage and composition. Nat Methods. 2014;11(11):1144-1146. https://doi. org/10.1038/nmeth.3103.

84. Wu Y-W, Tang Y-H, Tringe SG, Simmons BA, Singer SW: MaxBin: an automated binning method to recover individual genomes from metagenomes using an expectation-maximization algorithm. Microbiome. 2014;2(1). https://doi.org/10.1186/2049-2618-2-26.

85. Kang DD, Froula J, Egan R, Wang Z: MetaBAT, an efficient tool for accurately reconstructing single genomes from complex microbial communities. PeerJ. 2015;3. https://doi.org/10.7717/peeri.1165.

86. Sieber CMK, Probst AJ, Sharrar A, Thomas BC, Hess M, Tringe SG, Banfield JF: Recovery of genomes from metagenomes via a dereplication, aggregation and scoring strategy. Nat Microbiol. 2018;3(7):836-843. https://doi.org/10. 1038/s41564-018-0171-1.

87. Olm MR, Brown CT, Brooks B, Banfield JF: dRep: a tool for fast and accurate genomic comparisons that enables improved genome recovery from metagenomes through de-replication. ISME J. 2017;11(12):2864-2868. https://doi.org/10.1038/ismej.2017.126.

88. Parks DH, Imelfort M, Skennerton CT, Hugenholtz P, Tyson GW: CheckM: assessing the quality of microbial genomes recovered from isolates, single cells, and metagenomes. Genome Res. 2015;25(7):1043-1055. https://doi.org/ 10.1101/gr.186072.114

\section{Publisher's Note}

Springer Nature remains neutral with regard to jurisdictional claims in published maps and institutional affiliations. 